\title{
Neutralino-stop coannihilation into electroweak gauge and Higgs bosons at one loop
}

\author{
J. Harz, ${ }^{1, *}$ B. Herrmann, ${ }^{2, \dagger}$ M. Klasen, ${ }^{3,+}$ K. Kovař́ik, ${ }^{4, \S}$ and Q. Le Boulc' $h^{5, \|}$ \\ ${ }^{1}$ Deutsches Elektronen-Synchrotron (DESY), Notkestraße 85, D-22607 Hamburg, Germany \\ ${ }^{2}$ Laboratoire d'Annecy-le-Vieux de Physique Théorique (LAPTh), Université de Savoie/CNRS, \\ 9 Chemin de Bellevue, B.P. 110, F-74941 Annecy-le-Vieux, France \\ ${ }^{3}$ Institut für Theoretische Physik, Westfälische Wilhelms-Universität Münster, \\ Wilhelm-Klemm-Straße 9, D-48149 Münster, Germany \\ ${ }^{4}$ Institute for Theoretical Physics, Karlsruhe Institute of Technology, D-76128 Karlsruhe, Germany \\ ${ }^{5}$ Laboratoire de Physique Subatomique et de Cosmologie, Université Joseph Fourier/CNRS-IN2P3/INPG, \\ 53 Rue des Martyrs, F-38026 Grenoble, France \\ (Received 15 January 2013; published 25 March 2013)
}

\begin{abstract}
We compute the full $\mathrm{O}\left(\alpha_{s}\right)$ supersymmetric QCD corrections for neutralino-stop coannihilation into electroweak gauge and Higgs bosons in the minimal supersymmetric Standard Model. We show that these annihilation channels are phenomenologically relevant within the so-called phenomenological minimal supersymmetric Standard Model, in particular in the light of the observation of a Higgs-like particle with a mass of about $126 \mathrm{GeV}$ at the LHC. We present in detail our calculation, including the renormalization scheme, the infrared treatment, and the kinematical subtleties to be addressed. Numerical results for the coannihilation cross sections and the predicted neutralino relic density are presented. We demonstrate that the impact of including the corrections on the cosmologically preferred region of parameter space is larger than the current experimental uncertainty from WMAP data.
\end{abstract}

DOI: 10.1103/PhysRevD.87.054031

\section{INTRODUCTION}

Many astrophysical observations over a wide range of length scales provide today convincing evidence of a sizable cold dark matter (CDM) component in the Universe. The most recent measurements of the WMAP satellite in combination with baryonic acoustic oscillation and supernova data [1] constrain the dark matter relic density to the very precise value of

$$
\Omega_{\mathrm{CDM}} h^{2}=0.1126 \pm 0.0036
$$

at $1 \sigma$ confidence level, where $h$ denotes the present Hubble expansion rate $H_{0}$ in units of $100 \mathrm{~km} \mathrm{~s}^{-1} \mathrm{Mpc}^{-1}$. The leading candidate for dark matter is a weakly interacting massive particle. Unfortunately, the Standard Model does not contain any candidates that would be compatible with the properties of cold dark matter, the neutrinos being too light.

In contrast, the minimal supersymmetric Standard Model (MSSM) with conserved $R$ parity contains with the lightest neutralino, denoted $\tilde{\chi}_{1}^{0}$, a stable weakly interacting massive particle. Since supersymmetry (SUSY) offers many other theoretical and phenomenological advantages, the neutralino is by far the most studied dark matter candidate. The time evolution of its number density $n_{\chi}$ is described by the Boltzmann equation

\footnotetext{
*julia.harz@desy.de

†herrmann@lapth.cnrs.fr

¥michael.klasen@uni-muenster.de

${ }^{\S}$ kovarik@particle.uni-karlsruhe.de

"leboulch@lpsc.in2p3.fr
}

PACS numbers: 12.38.Bx, 12.60.Jv, 95.30.Cq, 95.35.+d

$$
\frac{\mathrm{d} n_{\chi}}{\mathrm{d} t}=-3 H n_{\chi}-\left\langle\sigma_{\mathrm{ann}} v\right\rangle\left[n_{\chi}^{2}-\left(n_{\chi}^{\mathrm{eq}}\right)^{2}\right]
$$

where the first term on the right-hand side containing the Hubble parameter $H$ stands for the dilution of dark matter due to the expansion of the Universe. The remaining terms reduce (increase) the number of dark matter particles by their annihilation (creation) in collisions with other particles. The term $\left\langle\sigma_{\mathrm{ann}} v\right\rangle$ denotes the thermal average of the annihilation cross section of the neutralino

$$
\begin{aligned}
\left\langle\sigma_{\mathrm{ann}} v\right\rangle & =\frac{\int \sigma v e^{-E_{1} / T} e^{-E_{2} / T} \mathrm{~d}^{3} p_{1} \mathrm{~d}^{3} p_{2}}{\int e^{-E_{1} / T} e^{-E_{2} / T} \mathrm{~d}^{3} p_{1} \mathrm{~d}^{3} p_{2}} \\
& =\frac{1}{8 m^{4} T K_{2}^{2}(m / T)} \int_{4 m^{2}}^{\infty} \sigma\left(s-4 m^{2}\right) \sqrt{s} K_{1}(\sqrt{s} / T) \mathrm{d} s,
\end{aligned}
$$

where $K_{i}$ are the modified Bessel functions of the second kind of order $i$ (for details see Ref. [2]).

Here we will consider the case when heavier, unstable supersymmetric particles survive in the Universe for sufficient time to affect the relic density of the dark matter particle. In this case, Eq. (1.2) has to be modified to account for the interactions between all particles and solve a system of Boltzmann equations for number densities $n_{i}$ for each surviving particle species,

$$
\frac{\mathrm{d} n_{i}}{\mathrm{~d} t}=-3 H n_{i}-\left\langle\sigma_{i j} v_{i j}\right\rangle\left(n_{i} n_{j}-n_{i}^{\mathrm{eq}} n_{j}^{\mathrm{eq}}\right),
$$

where $\sigma_{i j} \equiv \sigma\left(\chi_{i} \chi_{j} \rightarrow X\right)$ is the cross section of the annihilation of particle $i$ with particle $j$, and $v_{i j}$ is their 
relative velocity. As all heavier particles eventually decay into the dark matter particle, the relevant quantity is the total number density $n_{\chi}=\sum_{i} n_{i}$, and we can reformulate the problem into solving a single Boltzmann equation similar to Eq. (1.2) for the total number density with the annihilation cross section $\left\langle\sigma_{\mathrm{ann}} v\right\rangle$ replaced by an effective cross section $\left\langle\sigma_{\text {eff }} v\right\rangle$. More precisely, this cross section is given by

$$
\left\langle\sigma_{\mathrm{eff}} v\right\rangle=\sum_{i, j} \sigma_{i j} v_{i j} \frac{n_{i}^{\mathrm{eq}}}{n_{\chi}^{\mathrm{eq}}} \frac{n_{j}^{\mathrm{eq}}}{n_{\chi}^{\mathrm{eq}}},
$$

where the sum runs over all MSSM particles $i$ and $j$ (for a detailed discussion see Refs. [3,4]). The ratio of their respective number density in thermal equilibrium, $n_{i}^{\mathrm{eq}}$, and the number density of the neutralino, $n_{\chi}^{\mathrm{eq}}$, at the temperature $T$ is Boltzmann suppressed,

$$
\frac{n_{i}^{\mathrm{eq}}}{n_{\chi}^{\mathrm{eq}}} \sim \exp \left[-\frac{m_{i}-m_{\chi}}{T}\right],
$$

so that only particles, whose masses are almost degenerate with the one of lightest neutralino, can give sizable contributions. In the MSSM, typical examples of relevant coannihilations are those of the neutralino with the lightest slepton or squark, or with another gaugino. Moreover, pair annihilations of the next-to-lightest superpartner can be non-negligible. Having solved the Boltzmann equation numerically, the relic density is finally obtained through

$$
\Omega_{\chi} h^{2}=\frac{m_{\chi} n_{\chi}}{\rho_{\text {crit }}},
$$

where $n_{\chi}$ is the current number density of the neutralino and $\rho_{\text {crit }}$ is the critical density of the Universe. Comparing the predicted value obtained by solving the Boltzmann equation to the observational limits in Eq. (1.1) allows one to identify cosmologically (dis-)favored regions of the MSSM parameter space and thus to obtain important information that is complementary to collider searches and precision measurements.

The procedure described above is unfortunately subject to several uncertainties. The first source of uncertainty lies in the extraction of the relic density of CDM from cosmological data as given in Eq. (1.1). The extraction is based on a simple cosmological model, the $\Lambda \mathrm{CDM}$ model, which uses a minimal set of six parameters to fit the available cosmological data and bases its conclusions on the Standard Model of cosmology [1]. It has been shown that changing either the number of free parameters of the model used to fit the cosmological data [5] or modifying the assumptions contained in the Standard Model of cosmology (e.g., altering the expansion rate in the primordial universe or later, but still before big bang nucleosynthesis [6]), may change the extracted central value of $\Omega_{\mathrm{CDM}} h^{2}$ along with the confidence levels.
The second source of uncertainty in identifying (dis-) favored regions of the MSSM parameter space is connected to the calculation of the essential parameters such as masses and couplings of supersymmetric particles. As the relic density is very sensitive to the mass of the neutralino [see Eq. (1.7)], any uncertainty in the calculation of its mass directly translates into an uncertainty on the calculated relic density. Moreover, the relic density also strongly depends on the (co-)annihilation cross section, which in turn crucially depends on the masses of the remaining particles and their couplings to the neutralino. In the MSSM, the mass and the couplings of the neutralino as well as any other relevant couplings and masses are typically obtained using a dedicated spectrum calculator (see, e.g., Ref. [7]), which evolves all parameters down from a grand unification scale and calculates the masses and couplings for all particles at the weak scale. Different treatments of the radiative corrections for masses and couplings as well as different implementations of the renormalization group equations in various MSSM spectrum calculations can lead to differences in the predictions for the relic density and thus in the preferred or excluded regions of the MSSM parameter space (for details see, e.g., Ref. [8]).

The uncertainty which we will address in this paper does not fall into either of the above mentioned categories, but concerns the precision, with which the (co-) annihilation cross sections in Eq. (1.5) are computed. The cross sections in public dark matter tools such as DarkSUSY [9] or microOMEGAs [10] are implemented using only an effective tree-level calculation. It is, however, well known that higher-order corrections, particularly those involving the strong coupling constant, can have a sizable impact on such processes. The impact of next-to-leading-order corrections to neutralino annihilation on the neutralino relic density has been discussed in several previous analyses. SUSY-QCD corrections to neutralino pair annihilation into quark-antiquark pairs have been studied in Refs. [11-13], while the corresponding electroweak corrections have been evaluated in Refs. [14-16]. The authors of Refs. [15,16] have also discussed the case of coannihilation of a neutralino with another gaugino. Further studies rely on effective coupling approaches in order to capture certain classes of corrections to neutralino pair annihilation and coannihilation with a tau slepton $[17,18]$. All these analyses show that radiative corrections are not negligible in the context of relic density calculations, the impact of the corrections being larger than the experimental uncertainty from WMAP in many regions of parameter space. With the Planck satellite data providing more precise cosmological measurements in the very near future, it becomes even more pressing that theoretical predictions match the experimental precision.

The important case of SUSY-QCD corrections to coannihilation of a neutralino with a scalar top has so far only 
been considered in Ref. [19]. This study concerns the very specific cases of coannihilation of a binolike neutralino with a right-handed stop into a top quark and a gluon as well as into a bottom quark and a $W$ boson. However, depending on the considered region of parameter space, many other final states, including those with other electroweak gauge and Higgs bosons, can become dominant. Moreover, in realistic supersymmetric scenarios, helicity mixing in the stop sector is usually non-negligible, as is the mixing of bino, wino, and Higgsino components in the lightest neutralino, which strongly influences its couplings and preferred (co-)annihilation channels. Therefore, we extend in this paper the analysis of QCD and SUSYQCD corrections to coannihilation of a neutralino with a stop by computing the general case of neutralino-stop coannihilation into a quark and a Higgs or an electroweak vector boson. The paper is organized as follows: In Sec. II, we first discuss the phenomenology of neutralino-stop coannihilation in the MSSM. We then describe in detail the calculation of the radiative corrections to the relevant processes in Sec. III. Numerical results for annihilation cross sections and dark matter relic densities in typical MSSM benchmark scenarios are presented in Sec. IV, and our conclusions are given in Sec. V.

\section{PHENOMENOLOGY OF NEUTRALINO-STOP COANNIHILATION}

As discussed in Sec. I, the coannihilation of the next-tolightest supersymmetric particle (NLSP) with the lightest neutralino can in certain regions of the MSSM parameter space become dominant and lead to a relic density that is compatible with the observational limit of Eq. (1.1). A particularly important example of such a NLSP is the scalar top, whose chirality eigenstates can mix significantly, e.g., when the trilinear coupling $A_{t}$ becomes large, and which can then have a lower mass eigenstate that is almost mass degenerate with the lightest neutralino $[20,21]$.

There is ample motivation for a light scalar top. First, a light stop is a necessary ingredient to achieve electroweak baryogenesis in the MSSM [22]. Second, "natural" SUSY models $[23,24]$ require a light third generation of sfermions in order to reduce fine-tuning and stay compatible with experimental constraints at the same time. This is due to the fact that the mass degeneracy between the lightest neutralino and NLSP weakens the LHC exclusion potential on the third-generation squark masses, since this degeneracy results in events with soft jets $[25,26]$. Third, interpreting the new boson with a mass of about $126 \mathrm{GeV}$ observed recently at the LHC [27-29] as a light $C P$-even Higgs boson $\left(h^{0}\right)$ implies within the MSSM a particular choice of parameters in the stop and sbottom sector [30]. The reason is that in the MSSM the lightest Higgs boson mass receives a large contribution from a loop containing scalar tops. The leading contribution to the mass coming from this loop together with the tree-level contribution can be expressed as $[31,32]$

$$
\begin{aligned}
m_{h^{0}}^{2}= & m_{Z}^{2} \cos ^{2} 2 \beta+\frac{3 g^{2} m_{t}^{4}}{8 \pi^{2} m_{W}^{2}} \\
& \times\left[\log \frac{M_{\text {SUSY }}^{2}}{m_{t}^{2}}+\frac{X_{t}^{2}}{M_{\text {SUSY }}^{2}}\left(1-\frac{X_{t}^{2}}{12 M_{\text {SUSY }}^{2}}\right)\right],
\end{aligned}
$$

with $X_{t}=A_{t}-\mu / \tan \beta$ and $M_{\mathrm{SUSY}}=\sqrt{m_{\tilde{t}_{1}} m_{\tilde{t}_{2}}}$. The maximal contribution from stop mixing is then obtained for $\left|X_{t}\right| \sim \sqrt{6} M_{\text {SUSY }}$, which favors a sizable trilinear coupling $A_{t}$ and consequently a rather light stop.

At tree level, the coannihilation of a neutralino and a stop into final states containing a quark and an electroweak gauge or Higgs boson is mediated either by an $s$-channel quark, a $t$-channel squark, or a $u$-channel neutralino or chargino exchange. The corresponding Feynman diagrams are depicted in Fig. 1. These processes compete with all other possible (co-)annihilation channels of the lightest neutralino and in certain cases also with stop pair annihilation.

In order to quantify the relative importance of the processes in Fig. 1, we have performed a random scan in the phenomenological MSSM. In the following we describe the settings and discuss in detail the results of our scan. According to the supersymmetry parameter analysis convention [33] the soft-breaking parameters are defined at the scale $Q=1 \mathrm{TeV}$. We have made a few simplifying assumptions, which bring the number of parameters down to eight. In the squark sector, we use a common mass parameter $M_{\tilde{q}_{1,2}}$ for the squarks of the first and second generation, leaving the common mass parameter $M_{\tilde{q}_{3}}$ for the left- and right-handed squarks of the third generation independent. In contrast, the slepton sector is characterized by a single mass parameter $M_{\tilde{\ell}}$ for all three generations. All trilinear couplings are set to zero except for the $A_{t}$ in the stop sector, which enters our calculations through the relation $T_{t}=Y_{t} A_{t}$ with the top Yukawa coupling $Y_{t}$. All gaugino masses are defined through the bino mass parameter $M_{1}$. The wino and gluino masses are then fixed by the

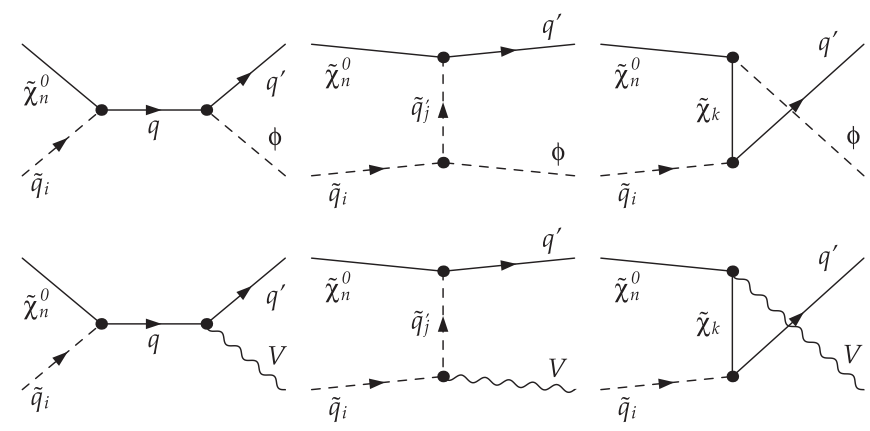

FIG. 1. Leading-order Feynman diagrams for neutralinosquark coannihilation into a quark and a Higgs boson ( $\left.\phi=h^{0}, H^{0}, A^{0}, H^{ \pm}\right)$or an electroweak gauge boson $(V=\gamma$, $\left.Z^{0}, W^{ \pm}\right)$. The $u$ channel is absent for a photon in the final state. 
relation $2 M_{1}=M_{2}=M_{3} / 3$, which is deduced from gaugino mass unification at the grand unified theory scale. Finally, the Higgs sector is specified by the pole mass $m_{A}$ of the pseudoscalar Higgs boson, the Higgsino mass parameter $\mu$, as well as the ratio $\tan \beta$ of the two vacuum expectation values of the Higgs doublets. In order to explore the parameter space, we have randomly generated 1.2 million parameter points within the following ranges for the eight input parameters:

$$
\begin{aligned}
500 \mathrm{GeV} \leq M_{\tilde{q}_{1,2}} & \leq 4000 \mathrm{GeV}, \\
100 \mathrm{GeV} \leq M_{\tilde{q}_{3}} & \leq 2500 \mathrm{GeV}, \\
500 \mathrm{GeV} \leq M_{\tilde{\ell}} & \leq 4000 \mathrm{GeV}, \\
\left|T_{t}\right| & \leq 5000 \mathrm{GeV}, \\
200 \mathrm{GeV} \leq M_{1} & \leq 1000 \mathrm{GeV}, \\
100 \mathrm{GeV} \leq m_{A} & \leq 2000 \mathrm{GeV}, \\
|\mu| & \leq 3000 \mathrm{GeV}, \\
2 \leq \tan \beta & \leq 50 .
\end{aligned}
$$

For each set of parameters, the physical mass spectrum and the related mixing matrices have been obtained using SPheno [7] (version 3.2.1). The neutralino relic density $\Omega_{\chi} h^{2}$ as well as the contributions from the individual (co-)annihilation channels have been computed using micrOMEGAs (version 2.4.1). For the numerical values of the Standard Model parameters we refer the reader to Ref. [34]. For a substantial number of these scenarios, coannihilation of the lightest neutralino with a scalar top plays an important role. This can be seen in the upper part of Fig. 2, where we show the relative contributions of the different final states channels to the total (co-)annihilation cross section as a function on the phenomenologically most relevant input parameters.

Experimentally viable scenarios have to satisfy a number of additional constraints. We therefore impose the following cuts on the neutralino relic density, the mass of the lightest Higgs boson, and the inclusive branching ratio of the most sensitive $B$-meson decay, $b \rightarrow s \gamma$ :

$$
\begin{aligned}
0.0946 & \leq \Omega_{\chi} h^{2} \leq 0.1306, \\
120 \mathrm{GeV} & \leq m_{h^{0}} \leq 130 \mathrm{GeV}, \\
2.77 \times 10^{-4} & \leq \mathrm{BR}(b \rightarrow s \gamma) \leq 4.33 \times 10^{-4} .
\end{aligned}
$$

The first cut selects the points which match the observed relic density of Eq. (1.1) within a $5 \sigma$ confidence interval. The second limit corresponds to a very conservative mass range for the new boson observed at the LHC [27,28]. Note that the theoretical uncertainty on the calculation of the lightest Higgs boson mass within SPheno is estimated to be about $3 \mathrm{GeV}$ [7]. Finally, the limits on the branching ratio of $b \rightarrow s \gamma$ correspond to a $3 \sigma$ interval around the observed value of $\mathrm{BR}(b \rightarrow s \gamma)=(3.55 \pm 0.26) \times 10^{-4}[35]$.

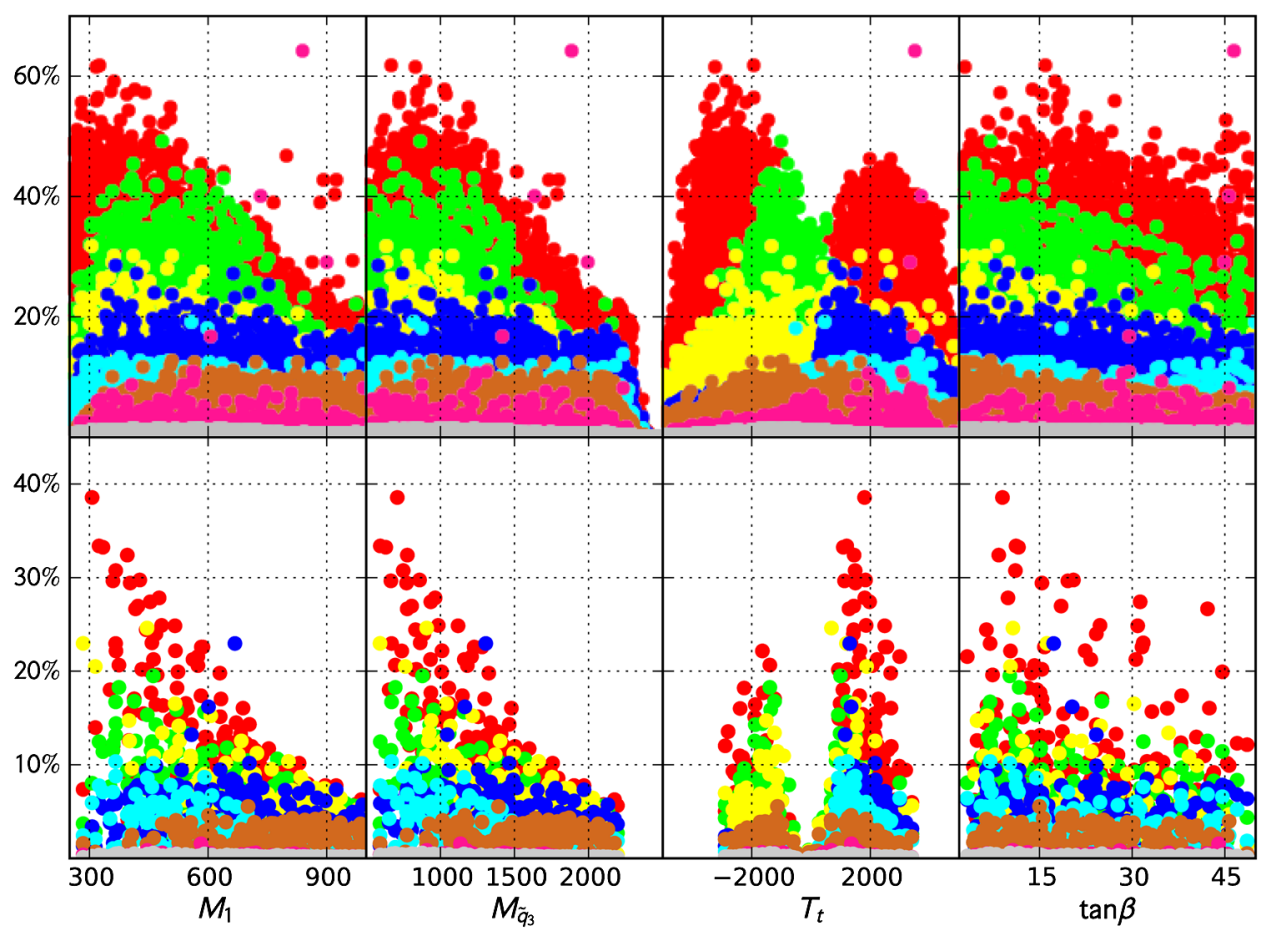

FIG. 2 (color online). Relative contributions of the neutralino-stop coannihilation channels for the generated parameter points as a function of the input parameters $M_{1}, M_{\tilde{q}_{3}}, T_{t}$, and $\tan \beta$ before (top) and after (bottom) applying the selection cuts of Eq. (2.3). Shown are the contributions from $t h^{0}$ (red), $t g$ (green), $t Z^{0}$ (blue), $t H^{0}$ (yellow), $b W^{+}$(cyan), $t A^{0}$ (brown), $b H^{+}$(pink), and $t \gamma$ (gray) final states. The parameters $M_{1}, M_{\tilde{q}_{3}}$, and $T_{t}$ are given in GeV. 
The points selected in this way are depicted in the lower part of Fig. 2, where we show again the relative contribution of the different neutralino-stop coannihilation channels. Applying the experimental cuts described above reduces the density of the points, but does not significantly change the shape of the distributions. As can be seen, the statistically most important final state is a top quark together with a light Higgs boson, followed by top quark and a gluon, a heavy $C P$-even Higgs boson, or a $Z$ boson. Comparable in size to the latter channel is the coannihilation into a bottom quark and a $W$ boson, whereas final states including a pseudoscalar Higgs boson, a charged Higgs boson, or a photon are less important.

One viable option how to satisfy the relic density bound and respect current exclusion limits from colliders is that the lightest neutralino and the lightest scalar top are almost mass degenerate. This is reflected in the left and left-center columns of Fig. 2 where we can observe a strikingly similar dependence of the fraction of coannihilation processes on the gaugino mass parameter $M_{1}$ and the third-generation squark mass parameter $M_{\tilde{q}_{3}}$, which are largely responsible for the masses of neutralinos and squarks of the third generation. For large values of both parameters, coannihilations cease to be important and annihilations of stops take their place as the dominating contribution of the total cross section.

In the right-center panel of Fig. 2 one can notice the interesting feature that after applying the cut on the lightest Higgs boson mass, large values of $T_{t}$ are preferred and the initially rather important percentage of top-gluon final states is reduced. This is driven by the fact that in contrast to the top-gluon final state, the Higgs boson mass prefers a sizable trilinear coupling. Moreover, positive values for $T_{t}$ are slightly preferred, since they allow a better maximization of the Higgs boson mass [30]. This is well visible in the center-right column of Fig. 2, where after applying the cuts two distinct ranges for the trilinear coupling parameter $T_{t}$ can be observed. These large values also enhance the Higgs-squark-squark coupling, which is present in the $t$ channel of the $t h^{0}$ final state. Accordingly, this changes the relative importance of the squark exchange with respect to the two other diagrams (quark or neutralino exchange, see Fig. 1). The $t$-channel enhancement also leads to an almost universal dominance of coannihilation into Higgs final states in the scenarios considered here. In other words, the same mechanism which drives the mass of the lightest Higgs boson to the observed value through important stop-loop contributions is responsible for the increase of neutralino-stop coannihilation into the lightest Higgs boson together with a top quark.

A similar connection between parameters that we mentioned above for $M_{1}$ and $M_{\tilde{q}_{3}}$ is found for $T_{t}$ and the Higgs parameter $\mu$. After the cuts, large and positive values of $\mu$ are preferred, which also enhances the Higgs-sfermionsfermion coupling mainly for the heavy $C P$-even Higgs with the same consequences as discussed above for large values of $T_{t}$.

The dependence on $\tan \beta$, on the other hand, is generally less pronounced. For coannihilation (mostly into top quarks), lower values of $\tan \beta$ are slightly preferred, since $b \bar{b}$ final states become more important for $\tan \beta \gtrsim 40$ [11]. The influence of the remaining input parameters, such as those related to first and second generation squarks, sbottoms, and sleptons, as well as the Higgsinos, is less important in this context. Therefore the corresponding dependencies are not displayed in Fig. 2.

For our numerical analysis, we have selected three characteristic scenarios, which we introduce and discuss in the following. They are listed in Table I and have been chosen in such a way that they represent qualitatively different scenarios (note, e.g., the differences in $M_{\tilde{q}_{1,2}}, m_{A}$, and $\mu$ ) and that they lead to different dominant coannihilation final states. As expected from Eq. (2.1), all three scenarios feature rather important trilinear coupling parameters $T_{t} \sim$ $1500-1800 \mathrm{GeV}$. The selected values of $\tan \beta$ are moderate, so that neutralino pair annihilation into bottom quarks is not important here. First and second generation squarks and sleptons are heavy compared to the stops in accordance with current LHC exclusion limits [36,37]. Moreover, the mass difference of the lightest neutralino and the scalar top is about $50 \mathrm{GeV}$ in each scenario and thus sufficiently small. In Table II we list the resulting values for the neutralino relic density, together with the contributions from the neutralino-stop coannihilation modes. These will be crucial to estimate the impact of our calculations on the final relic density.

Scenario I is characterized by the dominant coannihilation into a top quark and a light Higgs boson. Final states including a top quark and a $Z$ boson as well as a bottom quark and a $W$ boson contribute as well, but to a lesser

TABLE I. Three characteristic scenarios chosen in the phenomenological MSSM, which will be considered in this study. Given are the input parameters as described in the text, the lightest neutralino mass $m_{\tilde{\chi}_{1}^{0}}$, the lightest stop mass $m_{\tilde{t}_{1}}$, and the masses of the light and heavy $C P$-even Higgs bosons $m_{h^{0}}$ and $m_{H^{0}}$. All values except for $\tan \beta$ are given in GeV.

\begin{tabular}{|c|c|c|c|c|c|c|c|c|c|c|c|c|}
\hline & $M_{1}$ & $M_{\tilde{q}_{1,2}}$ & $M_{\tilde{q}_{3}}$ & $M_{\tilde{\ell}}$ & $T_{t}$ & $m_{A}$ & $\mu$ & $\tan \beta$ & $m_{\tilde{\chi}_{1}^{0}}$ & $m_{\tilde{t}_{1}}$ & $m_{h^{0}}$ & $m_{H^{0}}$ \\
\hline I & 306.9 & 2037.7 & 709.7 & 1499.3 & 1806.5 & 1495.6 & 2616.1 & 9.0 & 307.1 & 350.0 & 124.43 & 1530.72 \\
\hline II & 470.6 & 1261.2 & 905.3 & 1963.2 & 1514.8 & 1343.1 & 725.9 & 18.3 & 467.3 & 509.4 & 124.06 & 1342.77 \\
\hline III & 314.4 & 2870.5 & 763.6 & 2417.7 & 1877.5 & 386.0 & 2301.5 & 10.3 & 316.5 & 371.9 & 123.43 & 367.45 \\
\hline
\end{tabular}


TABLE II. Neutralino relic density and relative contributions of neutralino-stop coannihilation into a quark and a Higgs or electroweak gauge boson for the characteristic scenarios of Table I. The last column gives the sum of the listed contributions.

\begin{tabular}{lcccccc}
\hline \hline & $\Omega_{\chi} h^{2}$ & $\tilde{\chi}_{1}^{0} \tilde{t}_{1} \rightarrow t h^{0}$ & $\tilde{\chi}_{1}^{0} \tilde{t}_{1} \rightarrow t H^{0}$ & $\tilde{\chi}_{1}^{0} \tilde{t}_{1} \rightarrow t Z^{0}$ & $\tilde{\chi}_{1}^{0} \tilde{t}_{1} \rightarrow b W^{+}$ & Sum \\
\hline I & 0.114 & $38.5 \%$ & $\cdots$ & $3.4 \%$ & $5.9 \%$ & $47.8 \%$ \\
II & 0.116 & $24.6 \%$ & $\cdots$ & $10.7 \%$ & $3.4 \%$ & $38.7 \%$ \\
III & 0.111 & $14.2 \%$ & $20.7 \%$ & $1.2 \%$ & $2.1 \%$ & $38.2 \%$ \\
\hline \hline
\end{tabular}

extent. In total, neutralino-stop coannihilation with electroweak gauge and Higgs bosons final states accounts for almost half of the annihilation cross section at this example point. In order to understand which diagrams of Fig. 1 are most important in this context, we show in Fig. 3 the total tree-level cross sections of neutralino-stop coannihilation into the dominant final states for each characteristic scenario, together with the individual contributions of the different squared diagrams and interference terms. For the reasons discussed above, the exchange of a scalar top in the $t$ channel is the dominant mode at example point $\mathrm{I}$, followed by its interference with the exchange of a top quark in the $s$ channel (upper left plot). The squared $s$ channel is rather small, and all other channels are even negligible in this parameter configuration, so that they are not shown in Fig. 3.

In comparison to the first parameter point, scenario II has a smaller $\mu$ parameter, but a larger value of $\tan \beta$. Moreover, the gauginos and third-generation squarks are slightly heavier, and the trilinear coupling is slightly lower than for scenario I. As a consequence, the relative importance of the coannihilation channels is altered, as can be seen in Table II. In particular, the coannihilation into the lightest Higgs boson contributes less, allowing the final
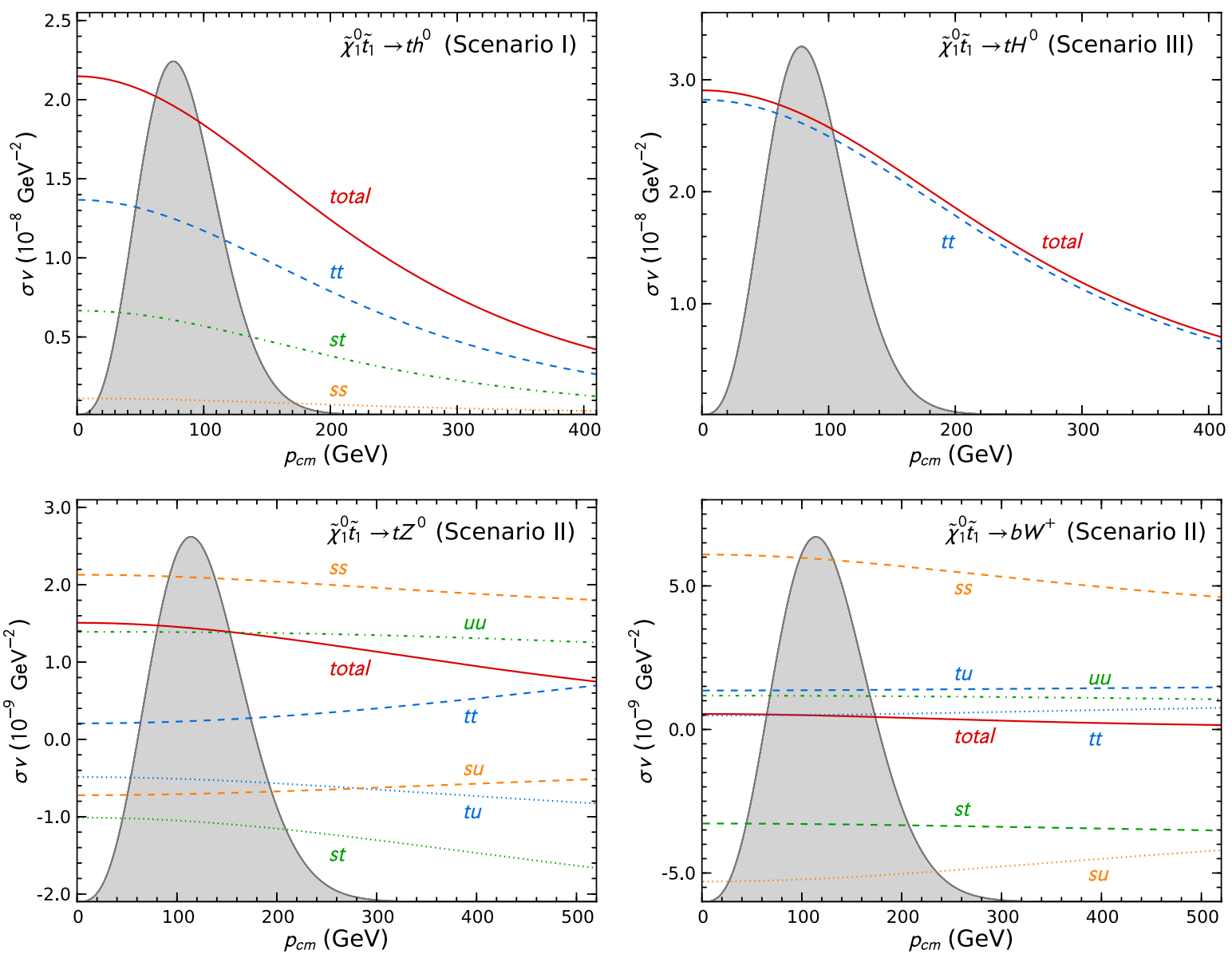

FIG. 3 (color online). Contribution of the different diagrams $(s-, t-$, and $u$-channel) depicted in Fig. 1. For the studied scenarios of Table I we show for selected coannihilation channels the tree-level cross section as well as the contribution of the different squared diagrams $(s s, t t, u u)$ and the interference terms $(s t, s u, t u)$. 
state containing a $Z$ boson to become more important. In contrast to the coannihilation into a Higgs boson, the dominant diagram in this case is the exchange of a top quark in the $s$ channel, as can be seen in Fig. 3 (lower left plot). For this scenario, we also show the individual contributions of the three diagrams for coannihilation into a bottom quark and a $W$ boson (lower right plot). As in the previous case, the $s$ channel is the dominant mode. Its absolute cross section value is even larger than for $t Z^{0}$ due to the larger phase space. However, large destructive interferences of this diagram with the subleading $t$ and $u$ channels decrease its cross section, so that the total value is almost an order of magnitude smaller than for the $Z$ boson.

Finally, scenario III is quite similar to scenario I with the exception of a very light pseudoscalar Higgs boson of $m_{A^{0}}=386 \mathrm{GeV}$. This leads to a similarly light heavy $C P$-even Higgs boson $H^{0}$ (see Table I). As a consequence, the coannihilation into heavy $C P$-even Higgs bosons in association with a top quark is now open and becomes the dominant contribution to neutralino-stop coannihilation (see Table II). The final state containing a light Higgs boson remains important, while coannihilations into $Z$ and $W$ bosons are marginal for this parameter point. As it was the case for the lightest Higgs boson, the coannihilation into $t H^{0}$ is dominated by the exchange of a scalar top in the $t$ channel (upper right plot of Fig. 3), which is again due to the enhanced trilinear coupling. The dominance is even more important here, which is explained by the modified mixing in the Higgs sector due to the smaller mass difference between $h^{0}$ and $H^{0}$.

\section{ONE-LOOP CROSS SECTIONS}

In this section, we turn to a detailed discussion of our analytical calculations of the full QCD and SUSY-QCD corrections to neutralino-stop coannihilation into electroweak gauge and Higgs bosons. We first describe the computation of the virtual loop corrections and the renormalization scheme employed in the quark and squark sector, then the analytical evaluation of real gluon emission diagrams and the corresponding cancellation of infrared singularities with those encountered in the virtual contributions. Finally, we address the subtle point of intermediate on-shell particles and how we subtract their resonant contributions.

\section{A. Virtual corrections and renormalization}

The coannihilation processes considered in this paper (see Fig. 1) include strongly interacting particles both in the initial and final states. As a consequence, the leading higher-order corrections to these processes come from loop diagrams containing a gluon, a gluino, a four-squark vertex, and from real radiation processes when a gluon is emitted from one of the squarks or quarks. The virtual corrections for the coannihilation processes contain propagator corrections, vertex corrections, and box diagrams. The corresponding diagrams are shown in Figs. 4-6, respectively. The divergences in these diagrams are regularized by performing the calculations in $D=4-2 \varepsilon$ dimensions. In order to preserve supersymmetry in the process, we use the dimensional reduction regularization

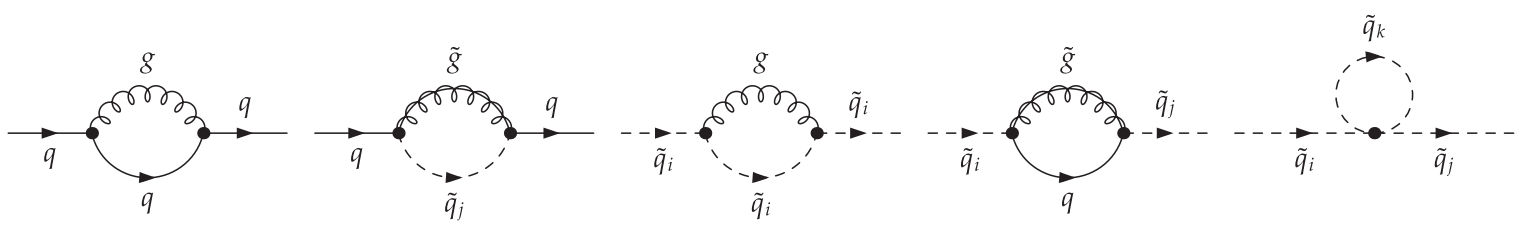

FIG. 4. Self-energy corrections for the quarks and squarks at one-loop level in QCD contributing to neutralino-squark coannihilation.
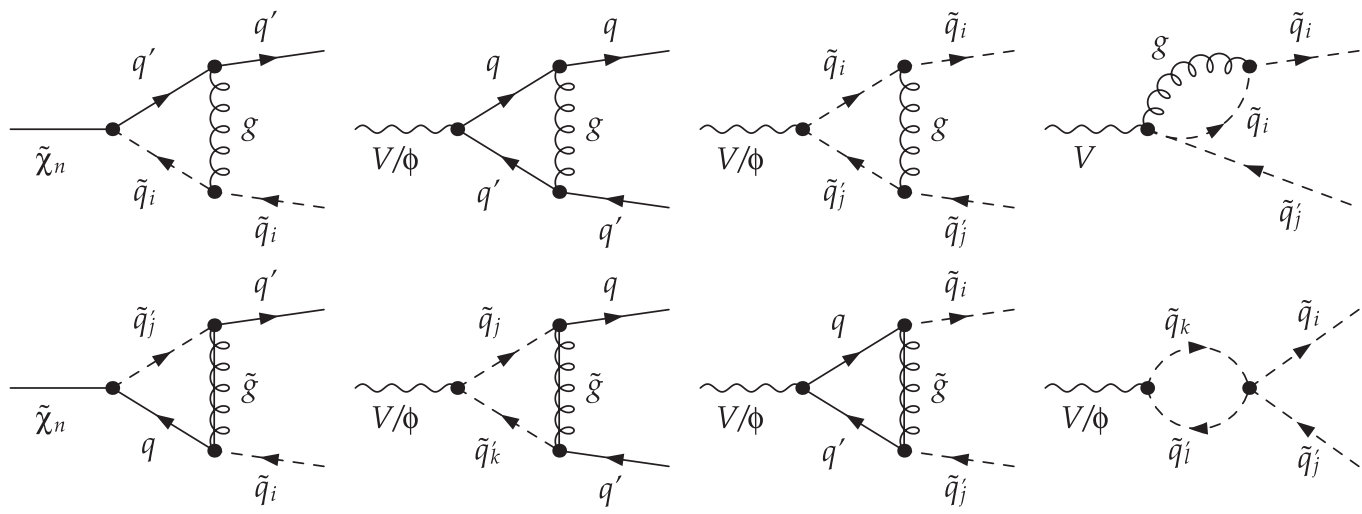

FIG. 5. Vertex corrections at one-loop level contributing to neutralino-squark coannihilation into quarks and Higgs $(\phi)$ or electroweak gauge $(V)$ bosons. The diagram involving the $V-g-\tilde{q}-\tilde{q}$ vertex is present only for the case of a gauge boson in the final state. 


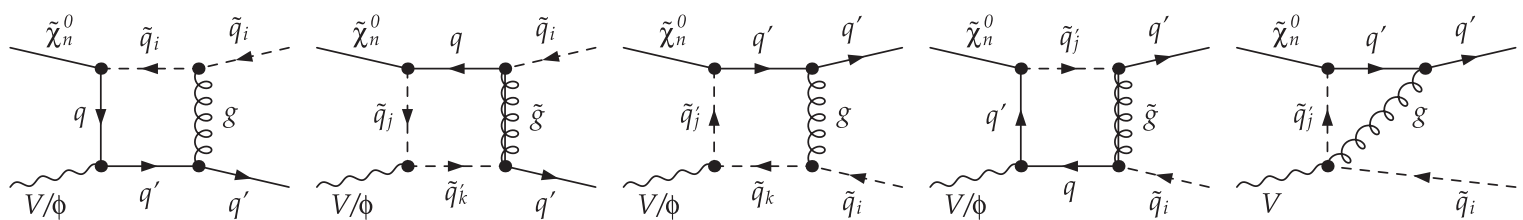

FIG. 6. Four-point diagrams at one-loop level contributing to neutralino-squark coannihilation into quarks and Higgs $(\phi)$ or electroweak gauge $(V)$ bosons. The last diagram involving the four-vertex is absent for a scalar in the final state.

scheme $(\overline{\mathrm{DR}})$. All tensor loop integrals are reduced using the standard Passarino-Veltman reduction [38]. The resulting scalar integrals are evaluated using the known results in, e.g., Refs. [39,40]. The renormalization and factorization scales are set to the center-of-mass energy $\sqrt{s}$. The amplitudes corresponding to the Feynman diagrams in Figs. 4-6 have been calculated analytically and crosschecked using the publicly available tools FeynArts [41], FeynCalc [42], and Form [43].

In order to cancel all arising ultraviolet (UV) singularities and render the cross section UV finite, we introduce counterterms to the relevant MSSM parameters and fields. When considering QCD and SUSY-QCD corrections to all processes that are needed to determine the neutralino relic density, a consistent treatment of all parameters in the quark and squark sector of the MSSM is essential. We

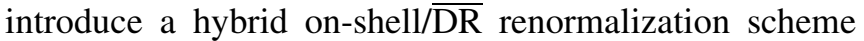
which is set up in such a way that it minimizes potential problems connected to sensitive parameters (e.g., the bottom trilinear coupling $A_{b}$ ) and is valid in a large region of MSSM parameter space. We will introduce all parameters and their treatment in detail below. We have explicitly verified that after renormalization all UV divergences cancel. The resulting expressions together with the renormalization scheme have been implemented in a numerical fortran code [44], which can serve as an extension to public dark matter tools such as DarkSUSY [9] and micrOMEGAs [10]. Similar renormalization schemes for the quark and squark sectors of the MSSM were already introduced and studied in Refs. $[45,46]$. Compared to those analyses, our approach differs significantly in the treatment of the squark mixing angles $\theta_{b}$ and $\theta_{t}$, but shares some important features with the RS2 scheme introduced in Ref. [46].

\section{Quark sector}

The process of neutralino-stop coannihilation considered here involves only quarks and squarks of the third generation. We will therefore discuss only the case of massive quarks. The parameters to be renormalized are the quark fields and masses. We perform the wave-function renormalization by introducing counterterms $\delta Z_{L, R}$ for each chirality of the third-generation quarks

$$
\left(\begin{array}{c}
q_{L} \\
q_{R}
\end{array}\right) \rightarrow\left(\begin{array}{cc}
1+\frac{1}{2} \delta Z_{L} & 0 \\
0 & 1+\frac{1}{2} \delta Z_{R}
\end{array}\right)\left(\begin{array}{l}
q_{L} \\
q_{R}
\end{array}\right)
$$

The wave-function renormalization constants are fixed by requiring the external quark propagators to have unit residue even at one-loop order. This leads to the following expression for the massive quarks $(q=t, b)$ :

$$
\begin{aligned}
\delta Z_{L}= & \Re\left\{-\Pi_{L}\left(m_{q}^{2}\right)-m_{q}^{2}\left[\dot{\Pi}_{L}\left(m_{q}^{2}\right)+\dot{\Pi}_{R}\left(m_{q}^{2}\right)\right]\right. \\
& +\frac{1}{2 m_{q}}\left[\Pi_{S L}\left(m_{q}^{2}\right)-\Pi_{S R}\left(m_{q}^{2}\right)\right] \\
& \left.-m_{q}\left[\dot{\Pi}_{S L}\left(m_{q}^{2}\right)+\dot{\Pi}_{S R}\left(m_{q}^{2}\right)\right]\right\}, \\
\delta Z_{R}= & \delta Z_{L}(L \leftrightarrow R),
\end{aligned}
$$

where $\Pi_{L, R}\left(k^{2}\right)$ and $\Pi_{S L, S R}\left(k^{2}\right)$ stand for the vector and the scalar parts of the two-point Green's function as defined in Ref. [47] and $\dot{\Pi}\left(m^{2}\right)=\left[\frac{\partial}{\partial k^{2}} \Pi\left(k^{2}\right)\right]_{k^{2}=m^{2}}$.

After the wave-function renormalization has been performed, we still have to renormalize the masses of the quarks. Although both the top and bottom quark are heavy, their properties are very different, and so is our treatment of their masses. On the one hand, the top quark does not form bound states and its physical mass is directly measurable. Therefore in our calculation, we use the physical (on-shell) top-quark mass $m_{t}=173.1 \mathrm{GeV}$. This implies using the on-shell mass counterterm for the top quark defined as

$\delta m_{t}=\frac{1}{2} \Re\left\{m_{t}\left[\Pi_{L}\left(m_{t}^{2}\right)+\Pi_{R}\left(m_{t}^{2}\right)\right]+\Pi_{S L}\left(m_{t}^{2}\right)+\Pi_{S R}\left(m_{t}^{2}\right)\right\}$.

On the other hand, the bottom quark forms hadrons and its mass cannot be directly measured. Conventionally a mass parameter $m_{b}\left(m_{b}\right)$ is extracted in the $\overline{\mathrm{MS}}$ renormalization scheme from the Standard Model analysis of $Y$ sum rules [48]. In order to obtain the appropriate bottom quark mass in the $\overline{\mathrm{DR}}$ renormalization scheme within the MSSM, we first use the Standard Model next-to-next-to-leadingorder renormalization group evolution to obtain the mass of the bottom quark at a scale $Q$ [49]. We then convert the $\overline{\mathrm{MS}}$ mass $m_{b}^{\overline{\mathrm{MS}}} \mathrm{S \textrm {SM }}(Q)$ to a mass in the $\overline{\mathrm{DR}}$ renormalization scheme $m_{b}^{\overline{\mathrm{DR}}, \mathrm{SM}}(Q)$ while still in the Standard Model [49]. Finally we apply the threshold corrections including also contributions from SUSY particles in the loop (denoted by $\Delta m_{b}$ ) 


$$
m_{b}^{\overline{\mathrm{DR}}, \mathrm{MSSM}}(Q)=m_{b}^{\overline{\mathrm{DR}}, \mathrm{SM}}(Q)-\Delta m_{b} .
$$

The corresponding counterterm contains the pole in $\varepsilon=(4-D) / 2$ and can be written as

$$
\frac{\delta m_{b}^{\overline{\mathrm{DR}}}}{m_{b}}=(-2) \frac{\alpha_{s} C_{F}}{4 \pi} \frac{c_{\varepsilon}}{\varepsilon},
$$

where we factored out the constant $c_{\varepsilon}=\Gamma(1+\varepsilon)(4 \pi)^{\varepsilon}$. One prominent place where the quark masses enter the calculation is through the Yukawa couplings of the Higgs bosons to the quarks. Especially the Yukawa couplings of the bottom quark were extensively studied in the decays of Higgs bosons in the Standard Model. Important QCD and top-quark induced corrections to the coupling of Higgs bosons to bottom quarks were calculated up to $\mathcal{O}\left(\alpha_{s}^{4}\right)$ [50] and can be used to define an effective Yukawa coupling which includes these corrections as

$$
\left[\left(h_{b}^{\overline{\mathrm{MS}}, \mathrm{QCD}, \Phi}\right)(Q)\right]^{2}=\left[\left(h_{b}^{\overline{\mathrm{MS}}, \Phi}\right)(Q)\right]^{2}\left[1+\Delta_{\mathrm{QCD}}+\Delta_{t}^{\Phi}\right],
$$

for each Higgs boson $\Phi=h^{0}, H^{0}, A^{0}$. The QCD corrections $\Delta_{\mathrm{QCD}}$ are explicitly given by

$$
\begin{aligned}
\Delta_{\mathrm{QCD}}= & \frac{\alpha_{s}(Q)}{\pi} C_{F} \frac{17}{4}+\frac{\alpha_{s}^{2}(Q)}{\pi^{2}}\left[35.94-1.359 n_{f}\right] \\
& +\frac{\alpha_{s}^{3}(Q)}{\pi^{3}}\left[164.14-25.77 n_{f}+0.259 n_{f}^{2}\right] \\
& +\frac{\alpha_{s}^{4}(Q)}{\pi^{4}}\left[39.34-220.9 n_{f}+9.685 n_{f}^{2}-0.0205 n_{f}^{3}\right],
\end{aligned}
$$

and the top-quark induced corrections $\Delta_{t}^{\Phi}$ for each Higgs boson $\Phi$ read

$$
\begin{gathered}
\Delta_{t}^{h}=c_{h}(Q)\left[1.57-\frac{2}{3} \log \frac{Q^{2}}{m_{t}^{2}}+\frac{1}{9} \log ^{2} \frac{m_{b}^{2}(Q)}{Q^{2}}\right], \\
\Delta_{t}^{H}=c_{H}(Q)\left[1.57-\frac{2}{3} \log \frac{Q^{2}}{m_{t}^{2}}+\frac{1}{9} \log ^{2} \frac{m_{b}^{2}(Q)}{Q^{2}}\right], \\
\Delta_{t}^{A}=c_{A}(Q)\left[\frac{23}{6}-\log \frac{Q^{2}}{m_{t}^{2}}+\frac{1}{6} \log ^{2} \frac{m_{b}^{2}(Q)}{Q^{2}}\right],
\end{gathered}
$$

with

$\left\{c_{h}(Q), c_{H}(Q), c_{A}(Q)\right\}=\frac{\alpha_{s}^{2}(Q)}{\pi^{2}}\left\{\frac{1}{\tan \alpha \tan \beta}, \frac{\tan \alpha}{\tan \beta}, \frac{1}{\tan ^{2} \beta}\right\}$.

We take into account these corrections excluding the oneloop part as it is provided consistently through our own calculation.
In the MSSM, the Yukawa coupling to bottom quarks can receive large corrections for large $\tan \beta$ or large $A_{b}$, even beyond the next-to-leading order, which can affect our analysis. Therefore, in addition, we include these corrections that can be resummed to all orders in perturbation theory $[51,52]$. Denoting the resummable part by $\Delta_{b}$ we redefine the bottom quark Yukawa couplings as

$$
\begin{gathered}
h_{b}^{\mathrm{MSSM}, h}(Q)=\frac{h_{b}^{\overline{\mathrm{MS}}, \mathrm{QCD}, h}(Q)}{1+\Delta_{b}}\left[1-\frac{\Delta_{b}}{\tan \alpha \tan \beta}\right], \\
h_{b}^{\mathrm{MSSM}, H}(Q)=\frac{h_{b}^{\overline{\mathrm{MS}}, \mathrm{QCD}, H}(Q)}{1+\Delta_{b}}\left[1+\Delta_{b} \frac{\tan \alpha}{\tan \beta}\right], \\
h_{b}^{\mathrm{MSSM}, A}(Q)=\frac{h_{b}^{\overline{\mathrm{MS}}, \mathrm{QCD}, A}(Q)}{1+\Delta_{b}}\left[1-\frac{\Delta_{b}}{\tan ^{2} \beta}\right] .
\end{gathered}
$$

In the same way as for the QCD corrections, we exclude the one-loop part of these SUSY-QCD corrections and include only the resummed remainder, since the one-loop part is already present in our calculation.

\section{Squark sector}

As in the above discussion for quarks, we will address here only the squarks of the third generation, i.e., stops and sbottoms. We work in the mass eigenstate basis and introduce the wave-function renormalization counterterms $\delta Z_{i j}$ through

$$
\tilde{q}_{i} \rightarrow\left(\delta_{i j}+\frac{1}{2} \delta Z_{i j}\right) \tilde{q}_{j},
$$

where in contrast to the case of quarks the $\delta Z_{i j}$ include also off-diagonal terms. The wave-function renormalization counterterms are again fixed by requiring that the squark propagators have unit residue also at one-loop level. In addition we require that mixing for on-shell squarks is absent. These conditions lead to the counterterms

$$
\begin{aligned}
& \delta Z_{i i}=-\Re\left[\dot{\Pi}_{i i}^{\tilde{q}}\left(m_{\tilde{q}_{i}}^{2}\right)\right], \\
& \delta Z_{i j}=\frac{2 \Re\left[\Pi_{i j}^{\tilde{q}}\left(m_{\tilde{q}_{j}}^{2}\right)\right]}{m_{\tilde{q}_{i}}^{2}-m_{\tilde{q}_{j}}^{2}}, \quad \text { for } i \neq j,
\end{aligned}
$$

where $\Pi_{i j}^{\tilde{q}}\left(k^{2}\right)$ are again the two-point Green's functions, this time for squarks.

The renormalization of the squark masses is complicated due to the mixing of squarks of the third generation. Therefore, it has to be discussed in conjunction with the renormalization of all other parameters in the squark sector appearing in the mass matrix. At tree level, the masses $m_{\tilde{q}_{i}}^{2}$ for stops and sbottoms are obtained by diagonalization of the mass matrix 


$$
U^{\tilde{q}}\left(\begin{array}{cc}
M_{\tilde{Q}}^{2}+\left(I_{q}^{3 L}-e_{q} s_{W}^{2}\right) \cos 2 \beta m_{Z}^{2}+m_{q}^{2} & m_{q}\left(A_{q}-\mu(\tan \beta)^{-2 I_{q}^{3 L}}\right) \\
m_{q}\left(A_{q}-\mu(\tan \beta)^{\left.-2 I_{q}^{3 L}\right)}\right. & M_{\{\tilde{U}, \tilde{D}\}}^{2}+e_{q} s_{W}^{2} \cos 2 \beta m_{Z}^{2}+m_{q}^{2}
\end{array}\right)\left(U^{\tilde{q}}\right)^{\dagger}=\left(\begin{array}{cc}
m_{\tilde{q}_{1}}^{2} & 0 \\
0 & m_{\tilde{q}_{2}}^{2}
\end{array}\right)
$$

where $e_{q}$ is the fractional charge of the squark in units of $e$, $s_{W}$ is the sine of weak mixing angle, $I_{q}^{3 L}$ is the weak isospin of the squark, and $U^{\tilde{q}}$ are the squark mixing matrices. As it is well known, we have to consider both the stop and the sbottom sector at the same time, since due to $S U(2)$ symmetry the mass matrices share a common soft-breaking parameter $M_{\tilde{O}}^{2}$ connecting the two sectors. In fact, out of the total set of eleven parameters $M_{\tilde{Q}}^{2}, M_{\tilde{U}}^{2}, M_{\tilde{D}}^{2}, A_{t}, A_{b}, \theta_{\tilde{t}}$, $\theta_{\tilde{b}}, m_{\tilde{t}_{1}}^{2}, m_{\tilde{t}_{2}}^{2}, m_{\tilde{b}_{1}}^{2}$, and $m_{\tilde{t}_{2}}^{2}$, only five are completely independent and can be considered as input parameters. Their counterterms can then be freely chosen. The remaining parameters are derived by requiring that Eq. (3.19) is valid even at one-loop order.

Here, we adopt a hybrid on-shell//DR renormalization scheme choosing as input the parameters $A_{t}, A_{b}, m_{\tilde{t}_{1}}^{2}, m_{\tilde{b}_{1}}^{2}$, and $m_{\tilde{b}_{2}}^{2}$, where the trilinear couplings $A_{t}, A_{b}$ are defined in the $\overline{\mathrm{DR}}$ renormalization scheme and all input masses are defined on-shell. This choice is motivated by the fact that we want to obtain a renormalization scheme which is applicable for all annihilation and coannihilation processes, where squarks play an important role. For example, as the coannihilation processes are extremely sensitive to the mass of the lightest stop and as this mass also plays an important role in the $t$-channel exchange of neutralino annihilations [13], we choose to include its mass in the input parameters. It is then crucial to take its physical or on-shell definition. Moreover, due to the appearance of the trilinear parameters $A_{t}, A_{b}$ in the important Higgs-squarksquark coupling in the coannihilation processes, it is a natural choice to include them in our input set as well.
Given the possible problems with the one-loop definition of the $A_{b}$ parameter widely discussed in the literature $[46,53,54]$, we choose to define both trilinear parameters in the $\overline{\mathrm{DR}}$ scheme. A different approach would be to define these parameters in the on-shell scheme, e.g., through the decay process of a squark into a squark and a Higgs boson [45]. This, however, would require a dedicated treatment of the infrared divergences arising in such a calculation.

Having explained above our choice of renormalization scheme, we must now specify the counterterms for the input parameters depending on their definition. The counterterms for the on-shell masses $m_{\tilde{t}_{1}}^{2}, m_{\tilde{b}_{1}}^{2}$, and $m_{\tilde{b}_{2}}^{2}$ are defined in the usual way as

$$
\delta m_{\tilde{q}_{i}}^{2}=\Re\left[\Pi_{i i}^{\tilde{q}}\left(m_{\tilde{q}_{i}}^{2}\right)\right] .
$$

The $\overline{\mathrm{DR}}$ counterterms of the trilinear parameters contain only the UV poles and can be given in terms of other $\overline{\mathrm{DR}}$ counterterms as

$$
\begin{aligned}
\delta A_{\tilde{q}}^{\overline{\mathrm{DR}}}= & \frac{1}{m_{q}}\left[U_{11}^{\tilde{q}} U_{12}^{\tilde{q}}\left(\delta m_{\tilde{q}_{1}}^{2}\right)^{\overline{\mathrm{DR}}}+U_{21}^{\tilde{q}} U_{22}^{\tilde{q}}\left(\delta m_{\tilde{q}_{2}}^{2}\right)^{\overline{\mathrm{DR}}}\right. \\
& +\left(U_{21}^{\tilde{q}} U_{12}^{\tilde{q}}+U_{11}^{\tilde{q}} U_{22}^{\tilde{q}}\right)\left(m_{\tilde{q}_{1}}^{2}-m_{\tilde{q}_{2}}^{2}\right) \delta \theta_{\tilde{q}}^{\overline{\mathrm{DR}}} \\
& \left.-\frac{\delta m_{q}^{\overline{\mathrm{DR}}}}{m_{q}}\left(U_{11}^{\tilde{q}} U_{12}^{\tilde{q}} m_{\tilde{q}_{1}}^{2}+U_{21}^{\tilde{q}} U_{22}^{\tilde{q}} m_{\tilde{q}_{2}}^{2}\right)\right] .
\end{aligned}
$$

The remaining $\overline{\mathrm{DR}}$ counterterms for squark masses and their mixing angle are given as [for $j \neq i$; for the quark mass counterterm see Eq. (3.6)]

$$
\begin{aligned}
\left(\delta m_{\tilde{q}_{i}}^{2}\right)^{\overline{\mathrm{DR}}}= & \frac{\alpha_{s} C_{F}}{4 \pi} \frac{c_{\varepsilon}}{\varepsilon}\left[\left(\left(U_{i 1}^{\tilde{q}}\right)^{2}-\left(U_{i 2}^{\tilde{q}}\right)^{2}\right)^{2} m_{\tilde{q}_{i}}^{2}-m_{\tilde{q}_{i}}^{2}+\left(U_{21}^{\tilde{q}} U_{11}^{\tilde{q}}-U_{22}^{\tilde{q}} U_{12}^{\tilde{q}}\right)^{2} m_{\tilde{q}_{j}}^{2}+8 m_{q} m_{\tilde{g}} U_{i 1}^{\tilde{q}} U_{i 2}^{\tilde{q}}-4 m_{\tilde{g}}^{2}-4 m_{q}^{2}\right], \\
\delta \theta_{\tilde{q}}^{\overline{\mathrm{DR}}}= & \frac{\alpha_{s} C_{F}}{4 \pi} \frac{c_{\varepsilon}}{\varepsilon} \frac{1}{\left(m_{\tilde{q}_{1}}^{2}-m_{\tilde{q}_{2}}^{2}\right)}\left[\left(U_{21}^{\tilde{q}} U_{11}^{\tilde{q}}-U_{22}^{\tilde{q}} U_{12}^{\tilde{q}}\right)\left(\left(\left(U_{11}^{\tilde{q}}\right)^{2}-\left(U_{12}^{\tilde{q}}\right)^{2}\right)^{2} m_{\tilde{q}_{1}}^{2}+\left(\left(U_{21}^{\tilde{q}}\right)^{2}-\left(U_{22}^{\tilde{q}}\right)^{2}\right)^{2} m_{\tilde{q}_{2}}^{2}\right)\right. \\
& \left.+4 m_{\tilde{g}} m_{q}\left(U_{11}^{\tilde{q}} U_{22}^{\tilde{q}}+U_{12}^{\tilde{q}} U_{21}^{\tilde{q}}\right)\right] .
\end{aligned}
$$

The values of the dependent parameters $M_{\tilde{Q}}^{2}, M_{\tilde{U}}^{2}, M_{\tilde{D}}^{2}$, $m_{\tilde{t}_{2}}^{2}, \theta_{\tilde{t}}$, and $\theta_{\tilde{b}}$ are determined using Eq. (3.19). For example, by taking a trace and a determinant of both sides of Eq. (3.19) for stops and sbottoms, we can relate the four parameters $M_{\tilde{Q}}^{2}, M_{\tilde{U}}^{2}, M_{\tilde{D}}^{2}$, and $m_{\tilde{t}_{2}}^{2}$ to the on-shell sfermion masses and the other parameters of the mass matrix such as $\mu$ or $\tan \beta$, which do not receive any QCD corrections and hence do not require renormalization. Having determined all mass parameters, we diagonalize the stop and sbottom mass matrices leading to the values of both mixing matri- ces. The eigenvalues are then the chosen on-shell masses and by construction the dependent mass $m_{\tilde{t}_{2}}^{2}$.

The counterterms of the dependent parameters are derived also from the defining Eq. (3.19). We do not give counterterms for $M_{\tilde{Q}}^{2}, M_{\tilde{U}}^{2}, M_{\tilde{D}}^{2}$ as they never appear in any vertex. Unlike in other analyses where the mixing angles are the input parameters and their counterterms are, e.g., given as a combination of wave-function renormalization constants [47], here both mixing angles $\theta_{\tilde{t}}$ and $\theta_{\tilde{b}}$ are dependent and have the counterterms 


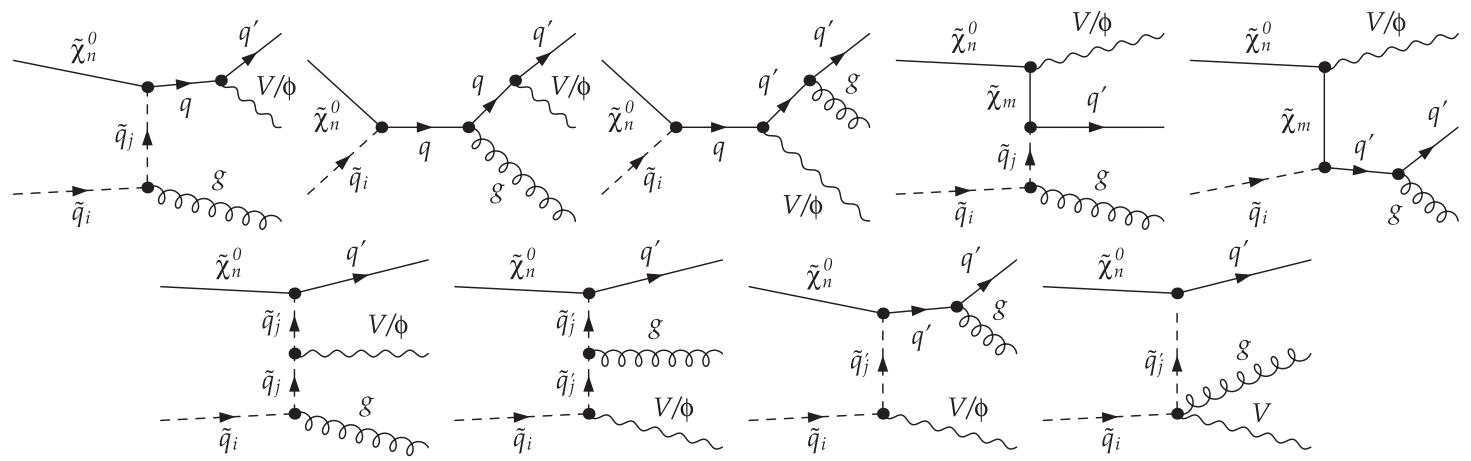

FIG. 7. Real gluon emission diagrams at one-loop level contributing to neutralino-squark coannihilation into quarks and Higgs ( $\phi$ ) or electroweak gauge $(V)$ bosons. The last diagram involving the four-vertex is absent for a scalar in the final state.

$$
\delta \theta_{\tilde{q}}=\frac{1}{\left(U_{21}^{\tilde{q}} U_{12}^{\tilde{q}}+U_{11}^{\tilde{q}} U_{22}^{\tilde{q}}\right)\left(m_{\tilde{q}_{1}}^{2}-m_{\tilde{q}_{2}}^{2}\right)}\left(\delta m_{q}\left(A_{q}-\mu(\tan \beta)^{-2 I_{q}^{3 L}}\right)+m_{q} \delta A_{q}-U_{11}^{\tilde{q}} U_{12}^{\tilde{q}}\left(\delta m_{\tilde{q}_{1}}^{2}-\delta m_{\tilde{q}_{2}}^{2}\right)\right)
$$

In the case of the stop mixing matrix this counterterm includes the last remaining undetermined counterterm of the mass of the heavy stop quark

$$
\begin{aligned}
\delta m_{\tilde{t}_{2}}^{2}= & \frac{1}{U_{21}^{\tilde{t}} U_{12}^{\tilde{t}}}\left[( U _ { 2 1 } ^ { \tilde { t } } U _ { 1 2 } ^ { \tilde { t } } + U _ { 1 1 } ^ { \tilde { t } } U _ { 2 2 } ^ { \tilde { t } } ) \left(\left(U_{11}^{\tilde{b}}\right)^{2} \delta m_{\tilde{b}_{1}}^{2}+\left(U_{21}^{\tilde{b}}\right)^{2} \delta m_{\tilde{b}_{2}}^{2}+2 U_{11}^{\tilde{b}} U_{21}^{\tilde{b}}\left(m_{\tilde{b}_{1}}^{2}-m_{\tilde{b}_{2}}^{2}\right) \delta \theta_{\tilde{b}}-2 m_{b} \delta m_{b}\right.\right. \\
& \left.\left.-\left(U_{11}^{\tilde{t}}\right)^{2} \delta m_{\tilde{t}_{1}}^{2}+2 m_{t} \delta m_{t}\right)-2 U_{11}^{\tilde{t}} U_{21}^{\tilde{t}}\left(\delta m_{t}\left(A_{t}-\mu / \tan \beta\right)+m_{t} \delta A_{t}-U_{11}^{\tilde{t}} U_{12}^{\tilde{t}} \delta m_{\tilde{t}_{1}}^{2}\right)\right] .
\end{aligned}
$$

This concludes our discussion of our renormalization scheme. We have discussed in detail the definition and renormalization of every relevant parameter in the quark and squark sector. By a clever choice of parameters we obtain a renormalization scheme which works in large parts of the relevant parameter space of the MSSM for all annihilation and coannihilation processes where quarks and squarks play an crucial role.

\section{B. Real corrections and infrared treatment}

Including only the virtual corrections with the renormalization constants does not lead to a finite result as some diagrams where a gluon is exchanged lead to a different type of divergence-the infrared (IR) divergence. These divergences cancel against similar divergences that come from the real radiation corrections where a gluon is emitted from one of the quarks or squarks, see diagrams in Fig. 7. The cancellation of these divergences is not as straightforward as in the case of ultraviolet divergences discussed above. It is because the IR divergence in the virtual diagrams can be explicitly isolated again by working in a general dimension $D$, whereas the divergence in the real corrections comes from the phase-space integration over the gluon phase space.

Several approaches exist in order to cancel these divergences, most notably the so-called phase-space slicing method [55-57] or the dipole subtraction method [58]. ${ }^{1}$ Here we use the phase-space slicing method which uses a lower cut on the gluon energy $\Delta E$ in the phase-space integration to render the real corrections finite. The missing divergent piece of the phase-space integral can be performed analytically in the limit of small energy of the gluon-the so-called soft-gluon approximation. Divergences obtained in the soft-gluon approximation then cancel analytically with those coming from the virtual corrections. In the soft-gluon approximation the phasespace integration factorizes as

$$
\left(\frac{\mathrm{d} \sigma}{\mathrm{d} \Omega}\right)_{\text {soft }}=F \times\left(\frac{\mathrm{d} \sigma}{\mathrm{d} \Omega}\right)_{\text {tree-level }},
$$

where $F$ contains the integral over the phase space of the gluon and therefore also the divergence. Explicitly, $F$ contains integrals of the form

$$
I_{a b}=\mu^{4-D} \int_{|\vec{k}| \leq \Delta E} \frac{\mathrm{d}^{D-1} k}{(2 \pi)^{D-4}} \frac{1}{k^{0}} \frac{(a \cdot b)}{(k \cdot a)(k \cdot b)},
$$

where $k$ is the 4-momentum of the gluon and $a$ and $b$ are 4-momenta of two external particles which can emit a gluon. These integrals are given in Refs. [57,59]. In our

\footnotetext{
${ }^{1}$ The implementation of a dedicated dipole subtraction method à la Catani-Seymour [58] is work in progress and subject to a later publication.
} 
case we use dimensional regularization to obtain an explicit form of the divergence.

The phase-space slicing method introduces a cut $\Delta E$ to separate the divergent part of the phase space. It appears in the original real corrections as a lower limit on the integration over the energy of the gluon and also explicitly in the cross section calculated in the soft-gluon approximation. In principle the dependence on this cut should completely vanish, but in practice the cancellation is limited by the stability of numerical integration of the real corrections. For practical purposes one has to choose a value for the cut such that it is small enough for the soft-gluon approximation to be valid in the region of phase-space given by $|\vec{k}| \leq \Delta E$, but at the same time large enough for the numerical integration of the real correction to be still possible. We have verified that in our calculation all cross sections are insensitive to the choice of this cut.

\section{On-shell propagators}

While including next-to-leading-order corrections to the studied neutralino coannihilation processes, we have to take care of a few subtleties. Some processes, although well defined and separate at tree level, cannot be unambiguously defined and separated when NLO corrections are considered. One such example is the process $\tilde{\chi}_{1}^{0} \tilde{t}_{1} \rightarrow b W$. Here, additional gluon radiation can be taken to be a real correction to the $W b$ process. However, it can equally well be considered to be neutralino-stop coannihilation with a gluon and a top quark in the final state where the top decays into a $W$ boson and a bottom quark. Despite the fact that these processes cannot be separated at NLO and one should strictly speaking include also their interference, for practical purposes it is desirable to find a way how to separate them.

Due to the above mentioned complication, one has to treat the process $\tilde{\chi}_{1}^{0} \tilde{t}_{1} \rightarrow b W g$ with care as it contains a top-quark propagator which can become on-shell. At tree level the large masses of the neutralino and the scalar top quark prevent the internal top quark to be on-shell. In contrast, when an additional gluon is radiated either from the initial stop or the internal top-quark propagator, the gluon can carry away enough energy for the top propagator to become on-shell. The relevant diagrams where this can occur are shown in Fig. 8. We regularize the appearing divergence from the on-shell propagator by introducing a

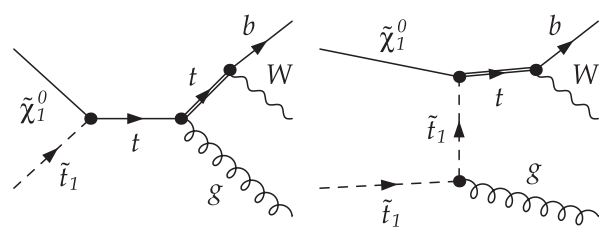

FIG. 8. Real gluon emission diagrams with a $W b$ final state where an internal top quark can become on shell, as indicated by a double line. width $\Gamma_{t}$ for the top quark in the problematic propagators, leading to a finite result for the integrated matrix elements for the real gluon emission. The matrix element when integrated over the whole phase space is very large as it includes also the leading-order coannihilation process $\tilde{\chi}_{1}^{0} \tilde{t}_{1} \rightarrow \operatorname{tg}$ with the top quark decaying into $W^{+} b$. This process is, however, already accounted for in the calculation of the neutralino relic density. To avoid double counting, we need to separate the two processes.

In order to treat the double counting in the real correction contribution, we use a local on-shell subtraction scheme [60-62], in which a locally gauge invariant term is subtracted from the original cross section that has been regularized as discussed above. The subtraction term is defined as the squared resonant amplitude with the top quark being on shell, except for the propagator denominator, which is kept as a general Breit-Wigner function

$$
\left|\mathcal{M}_{2 \rightarrow 3}^{\text {sub }}\right|^{2}=\frac{m_{t}^{2} \Gamma_{t}^{2}}{\left(p_{t}^{2}-m_{t}^{2}\right)^{2}+m_{t}^{2} \Gamma_{t}^{2}}\left|\mathcal{M}_{2 \rightarrow 3}^{\text {res }}\right|_{p_{t}^{2}=m_{t}^{2}}^{2}
$$

When the top quark is exactly on shell, the subtraction term is equal to the full $2 \rightarrow 3$ matrix element, while it decreases as a Breit-Wigner distribution when the top quark moves away from its pole. This method has the advantage that the resulting cross section retains the nonresonant interferences of the two processes. We have checked that the total cross section after subtraction is independent of the top-quark width.

Other diagrams with different final states can also include on-shell propagators but for most of them only in very specific configurations, e.g., mass degeneracy between $\tilde{t}_{1}$ and $\tilde{t}_{2}$ or between $\tilde{t}_{1}$ and $\tilde{b}_{1}$. Those cases are not relevant for our study of $\tilde{\chi}_{1}^{0} \tilde{t}_{1}$ coannihilation.

Another numerical instability arises from the fact that, in case of coannihilation into quark and photon, also the external photon of the real emission subprocess $\tilde{\chi}_{n}^{0} \tilde{q}_{i} \rightarrow$ $q g \gamma$ may become soft in certain regions of phase space, rendering the numerical integration unreliable. This issue can be addressed by introducing a cutoff on the photon energy in order to exclude the corresponding part of the phase space. This soft behavior (and the associated cut-off dependence) would vanish when including also electroweak corrections, which is, however, beyond the scope of this work. Moreover, as we have seen in Sec. II, the impact of this process in the scenarios considered in the present work is negligible.

\section{Numerical results}

Let us now discuss in detail the impact of the one-loop corrections on the coannihilation cross sections in our three scenarios of Table I. We have calculated radiative corrections to two types of processes, one with a Higgs boson and one with a vector boson in the final state. We have seen that at tree level the processes with the Higgs boson final state are dominated by a $t$-channel stop exchange, whereas the 

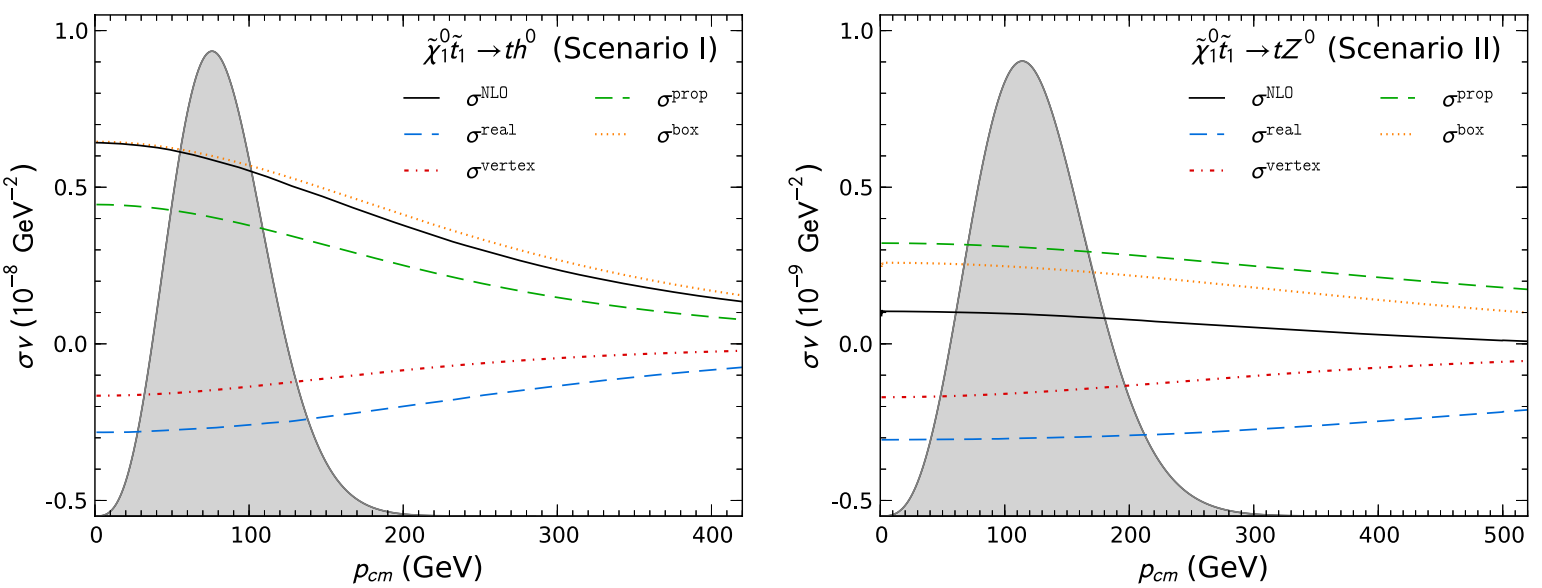

FIG. 9 (color online). Contribution of the different corrections to the total next-to-leading-order correction for the case of coannihilation into $t h^{0}$ for scenario I and into $t Z^{0}$ for scenario II. The real contribution $\sigma^{\text {real }}$ is defined as the sum of the hard radiation and the soft-gluon part with a cut on the gluon energy of $\Delta E=10^{-3} \sqrt{s}$. The gray area indicates the thermal distribution (in arbitrary units).

processes with a gauge vector boson are a mixture of all possible contributions (see Fig. 3).

These different compositions of the cross sections influence also the impact of various types of loop corrections which are displayed in Fig. 9. This figure shows a break down of the total next-to-leading correction to the cross section $\sigma v$ (without the tree-level contribution) into several UV finite contributions for both types of processes, $\tilde{\chi}_{1}^{0} \tilde{t}_{1} \rightarrow t h^{0}$ (scenario I) and $\tilde{\chi}_{1}^{0} \tilde{t}_{1} \rightarrow t Z^{0}$ (scenario II). Even though all contributions are UV finite, the box, vertex and real part of the correction are still IR divergent. This leads to a certain ambiguity in their exact definition. Each contribution contains an uncancelled pole along with an uncancelled logarithm of the large factorization scale. These large logarithms cause the box contribution to be artificially large and drive the real corrections (which in our case is a sum of the soft-gluon part and the hard radiation) to be negative.

Comparing the different loop contributions for the scalar and vector boson final states, one notices that the box and propagator corrections in the case of the Higgs boson final states are enhanced. This can be traced back to the fact that the cross section with a Higgs boson in the final state is dominated by the $t$-channel exchange. One of the loop corrections to the $t$ channel entails a correction to the stop propagator and a box diagram where a gluon is exchanged between the final state quark and the initial state squark. The enhanced box and propagator corrections lead to a large overall NLO correction in the case of the coannihilation cross section with the Higgs boson.

We show the cross sections of the respectively most relevant channel in each scenario in Fig. 10 and compare our tree-level calculation, the effective tree-level calculation implemented in micrOMEGAs and our full one-loop calculation. The upper parts show the cross sections $\sigma v$, while the lower panels show the ratio between the different cross sections.

For scenario I, where we show the channel $\tilde{\chi}_{1}^{0} \tilde{t}_{1} \rightarrow t h^{0}$, we have numerical agreement between our tree level and the micrOMEGAs calculation. The one-loop contributions increase the cross section by about $30 \%$ caused by the large contribution from the box diagrams and propagator corrections as discussed above. We observe a similar behavior for scenario III, where the final state with a heavy Higgs boson $H^{0}$ is dominant. Here, the one-loop cross section lies about $18 \%-20 \%$ above the tree levels, which again agree well among each other.

In case of coannihilation into a quark and an electroweak gauge boson, there is a few percent difference between our tree level and the one provided by micrOMEGAs. This difference stems from the fact that both tree levels use different parameters. Our tree level uses input parameters defined through the renormalization scheme discussed in detail in Sec. III A. It differs in several points from the parameters used by micrOMEGAs. More precisely, the shift between the two tree levels is largely due to a different definition of the squark mixing angles, which enter the calculation through the different interactions between squarks and quarks, e.g., the neutralino-squark-quark vertex.

The different influence of various definitions of the mixing angle on the two classes of processes we have calculated can be understood as follows: In the case of the Higgs boson final state, which is dominated by a squark exchange in the $t$ channel, the mixing angle $\theta_{\tilde{t}}$ enters the squark-squark-Higgs and the neutralino-squark-quark vertices. The internal propagator has to be summed over the two possible squark mass eigenstates, $\tilde{t}_{1}$ and $\tilde{t}_{2}$, making the result less sensitive to the exact value of the mixing angle. For the $s$-channel dominated coannihilation into $t Z^{0}$ or $b W^{+}$, the situation is quite different. Here, the mixing 

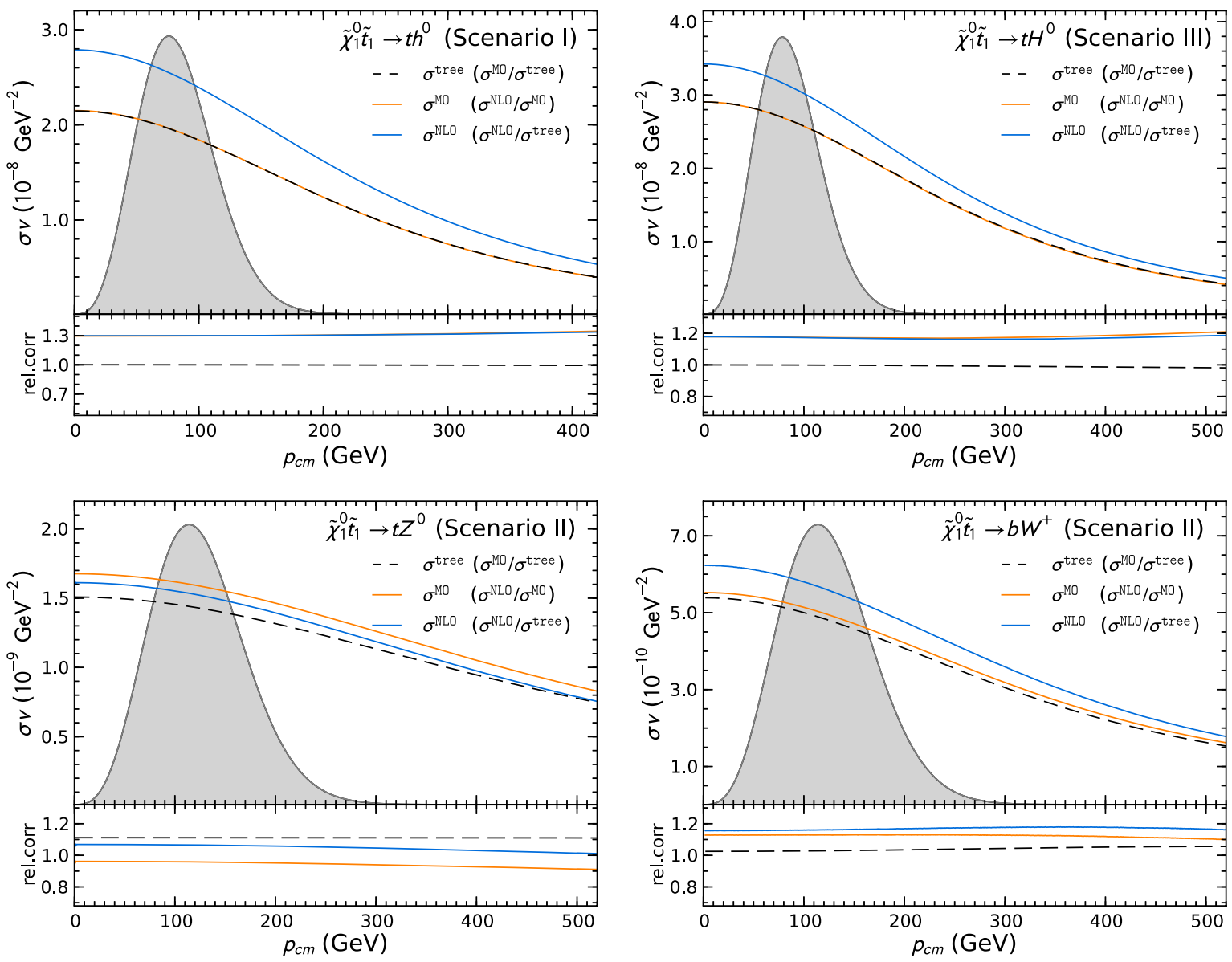

FIG. 10 (color online). Tree-level (black dashed line), full one-loop (dark blue solid line) and micrOMEGAs (light orange solid line) cross sections for selected coannihilation channels in the scenarios of Table I. The upper part of each plot shows the absolute value of $\sigma v$ together with the thermal distribution (in arbitrary units), whereas the lower part shows the corresponding relative shifts (second item in the legend).

angle appears in a single neutralino-squark-quark vertex, where the external squark is "fixed" to be $\tilde{t}_{1}$. The corresponding matrix element is therefore rather sensitive to changes in the mixing angle, which explains the observed difference between the two tree-level curves.

\section{IMPACT ON THE NEUTRALINO RELIC DENSITY}

The main purpose of this analysis is to investigate the impact of higher-order corrections on the neutralino relic density.

Our numerical implementation of the calculation described in Sec. III is used as an extension to the public package micrOMEGAs in order to evaluate the effect of the one-loop corrections on the neutralino relic density. We stress that our implementation is general so that it can be used for any neutralino-sfermion coannihilation process, even if we focus in this study on the case of $\tilde{\chi}_{1}^{0} \tilde{t}_{1}$, which is the most relevant process of this kind within the MSSM. Our numerical code is linked to micrOMEGAs in such a way that all relevant parameters, i.e., the masses and mixings of all particles, are passed between the two codes in a consistent way. In particular, we use SPheno to compute the supersymmetric mass spectrum for our characteristic scenarios as described in Sec. II.

In this section we compare the neutralino relic density obtained from the three different cross section calculations, which have been described in Sec. III: the one used by default in micrOMEGAs, evaluated by CalcHEP [63] at tree level, our cross section at tree level, and our calculation including the full next-to-leading-order SUSY-QCD corrections. The impact of the corrections compared to the tree-level results is studied for the three scenarios defined in Table I.

First, we focus on scenario I. We study the change of the relic density when a single input parameter is varied around our scenario I. In Fig. 11, we show $\Omega_{\chi} h^{2}$ as a function of the bino mass parameter $M_{1}$ and the trilinear coupling parameter $T_{t}$, calculated on the basis of the aforementioned three calculations for the neutralino-stop coannihilation. It is clearly visible that the relic density is 

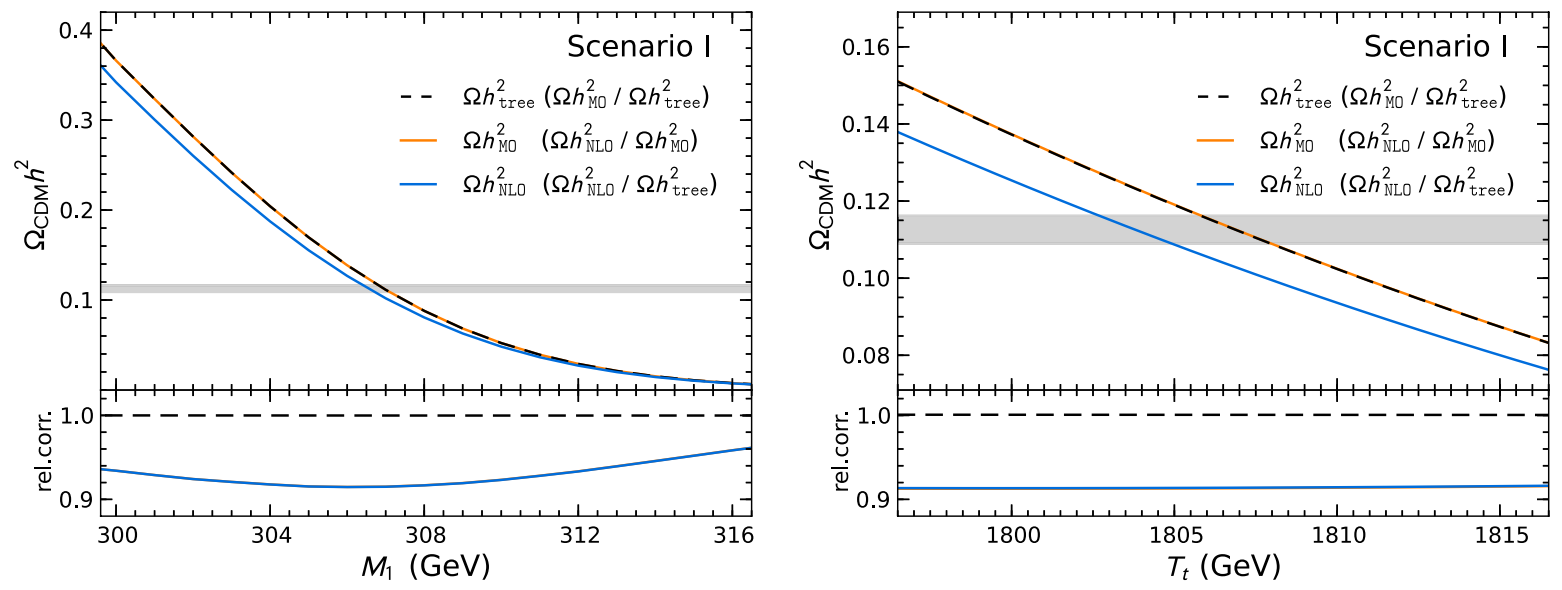

FIG. 11 (color online). The neutralino relic density $\Omega_{\chi} h^{2}$ as a function of $M_{1}$ (left) and $T_{t}$ (right) in our scenario I calculated using different coannihilation cross sections: default micrOMEGAs (light orange solid line), tree-level (black dashed line), and full one-loop (dark blue solid line). The gray band indicates the favored range according to Eq. (1.1). The lower part shows the relative impact of the one-loop correction on the relic density compared to the tree-level calculation (second item in the legend).

very sensitive to variations of the bino mass parameter. For higher values of $M_{1}$ the predicted relic density decreases rapidly due to a smaller mass splitting between the lightest neutralino and the lightest stop, which enhances the neutralino-stop coannihilation and in addition the stop-stop annihilation. In contrast, slightly lower values for the bino mass parameter increase the mass difference and suppress the contribution of coannihilation processes in favor of neutralino-neutralino annihilation. The predicted relic density is then higher due to the absence of coannihilation. Within the area which is favored by the measurements of WMAP, where the studied neutralino-stop coannihilation is dominant, a clear shift of the predicted relic density is visible when going from the default value calculated by micrOMEGAs to the one calculated using our full next-to-leading-order result.

The impact of the presented SUSY-QCD corrections to the given neutralino-stop coannihilation processes is even better visible in the lower part of Fig. 11, where we show the relative correction, i.e., the ratio of the relic density calculated with our full one-loop coannihilation cross section to the one included by default in micrOMEGAs and our tree level, respectively. For scenario I, our calculations result in a relative correction of about $9 \%$. This can be explained by the lightest Higgs final state, which has a contribution of around $38.5 \%$ to the total (co-)annihilation cross section with a corresponding correction of around $30 \%$ (see Fig. 10). With the current experimental uncertainty of about $3 \%$ according to Eq. (1.1), the impact of the presented corrections is significant and thus important to be taken into account.

The relic density is less sensitive to varying the trilinear coupling parameter $T_{t}$ around the value in scenario I $\left(T_{t}=1806.5 \mathrm{GeV}\right)$. This is depicted on the right-hand side of Fig. 11. Here, the difference between the uncorrected and corrected relic density in the cosmologically favored region corresponds to a difference of $3 \mathrm{GeV}$ in the parameter $T_{t}$.

One can infer more about the impact of the full next-toleading-order corrections in scenario I when looking at the first row of Fig. 13. On the left, the WMAP-favored region is shown as a function of two parameters-the mass parameter of the third generation of squarks $M_{\tilde{q}_{3}}$ and the bino mass parameter $M_{1}$. In the same plot solid black contour lines denote the relative impact of our correction to the default micrOMEGAs relic density. As the coannihilation into the lightest Higgs is the dominant contribution to the total (co-)annihilation cross section around the WMAP-favored region in this scenario, and as it receives large corrections, a relative correction of up to $9 \%$ on the relic density is observed. The correction is larger than current experimental uncertainties, which results in two separated WMAP-favored $1 \sigma$ bands corresponding to the default micrOMEGAs calculation (orange) and our full one-loop SUSY-QCD calculation (blue).

The cosmologically allowed band follows a straight line in the $M_{1}-M_{\tilde{q}_{3}}$ plane corresponding to a constant mass difference between the lightest neutralino and the lightest stop of about $40 \mathrm{GeV}$. Above this band where the neutralino becomes heavier and the mass difference decreases, the stop-stop annihilation becomes dominant. As it has typically a significant higher cross section than the coannihilation, it leads to a relic density which is too small. For large values of $M_{1}$ (in the gray area in the upper left corner) the stop becomes the lightest supersymmetric particle, which is disfavored as a suitable dark matter candidate both for its electric and color charge.

In the opposite direction, below the allowed band, the neutralino-stop and stop-stop (co-)annihilation are Boltzmann suppressed by a larger mass difference and neutralino annihilation becomes dominant. However, it 
has a lower cross section, such that the relic density becomes too big.

To conclude our analysis of scenario I, on the right plot in Fig. 13 we show WMAP preferred regions in the $\left(T_{t}, M_{1}\right)$ plane. Again, a clear separation of the two bands is visible, together with the small dependence on the trilinear coupling parameter $T_{t}$ (as already discussed for Fig. 11). In different green colors, the mass difference between the lightest and next-to-lightest supersymmetric particle is depicted supporting the claim that the cosmologically favored region follows a contour of a constant mass difference around 40-45 GeV. The solid black lines show the mass of the lightest Higgs boson in the selected parameter space. One can see that the whole WMAPfavored region in this plot lies within the recent Higgs mass limit $125.2 \mathrm{GeV} \pm 0.9 \mathrm{GeV}$ as reported in Ref. [29]. It is also interesting to note that the cosmological constraints from WMAP are at the moment more stringent than the current bounds on a Higgs-like particle.

Let us now focus on scenario II, which differs in several crucial features from the previously analyzed scenario I. One example is that the total coannihilation cross section has two dominating contributions from coannihilation into the lightest Higgs and into the $Z$ boson. In Fig. 12 we show separately the effect of SUSY-QCD corrections to each of the two dominant processes as a function of the parameters $M_{\tilde{q}_{3}}$ and $T_{t}$. One can see distinctly different effects higherorder corrections have on each process. As in scenario I, large corrections to coannihilation into the lightest Higgs bosons lead to a change of up to $6 \%$ in the relic density even though its relative importance in the total cross section dropped to $24 \%$ compared to scenario I. On the other hand corrections to coannihilation into the $Z$ boson are small (see Fig. 10) and also differ in sign. This leads to a reduction of the impact of SUSY-QCD corrections on the

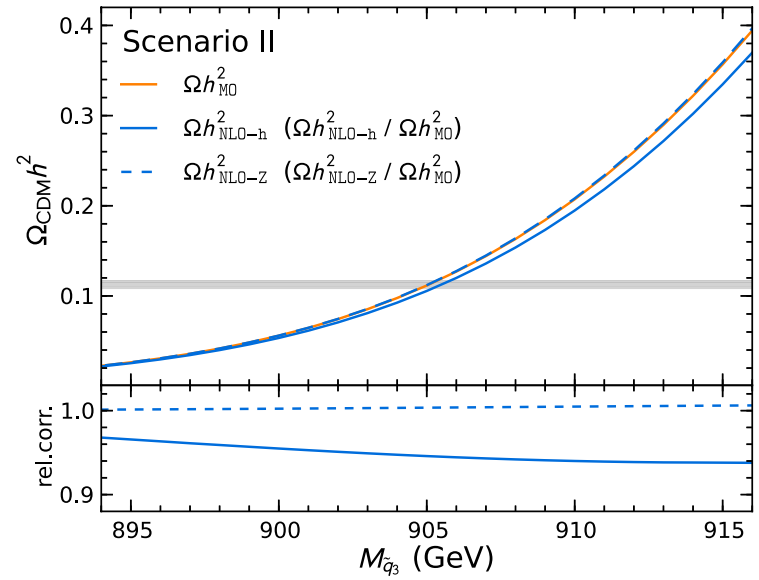

relic density in scenario II. The consequences can be seen in the second row of Fig. 13. One sees that due to the smaller correction of about 5\%-6\%, the two bands corresponding to the original micrOMEGAs relic density (orange) and the one obtained including our SUSY-QCD corrections (blue) overlap.

Scenario II is different from the others also in that the preferred WMAP region lies outside of the area with maximal coannihilation fraction. This is a direct consequence of the importance of the coannihilation into the $Z$ boson which has a smaller cross section and so in total coannihilation is not efficient enough to bring the relic density down to the level measured by WMAP (the allowed region receives sizable contributions from the stop annihilations).

In contrast to other scenarios, in scenario II coannihilation dominates in a region where the mass difference between the stop and the lightest neutralino is larger (about $70 \mathrm{GeV}$ ). This can be traced back to the masses of the lightest neutralino and the stop, which are much heavier than in the other two scenarios. As a result the freeze-out temperature, which is proportional to the mass of the dark matter particle, is higher. This means that the same Boltzmann suppression which for scenario I was obtained for a mass difference $40-45 \mathrm{GeV}$, is now reached for a larger mass splitting of $70 \mathrm{GeV}$.

In the third scenario, the light $C P$-even Higgs boson is the dominant contribution to neutralino-stop coannihilation and the characteristics of the plots in Fig. 13 are similar to scenario I. As the correction to the top- $H^{0}$ final states is not as large as for the top- $h^{0}$ final state in this example point (see Fig. 10), the overall impact on the relic density is thus smaller than for scenario I. A relative correction between $5 \%$ to $6 \%$ is reached. Nevertheless, a shift from the WMAP-favored region calculated by

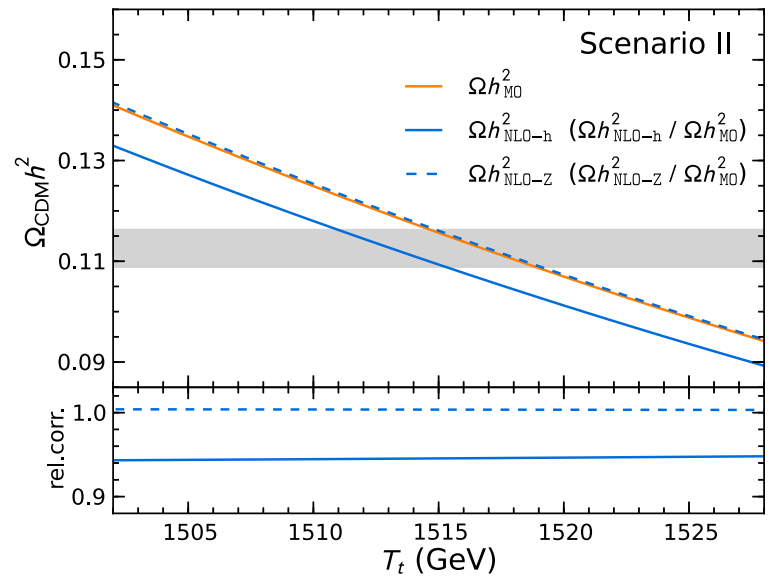

FIG. 12 (color online). The neutralino relic density $\Omega_{\chi} h^{2}$ as a function of $M_{\tilde{q}_{3}}$ (left) and $T_{t}$ (right) in our scenario II calculated using different coannihilation cross sections: default micrOMEGAs (light orange solid line), one-loop correction only for the $t h^{0}$ final state (dark blue solid line), and one-loop correction only for the $t Z^{0}$ final state (blue dashed line). The gray band indicates the favored range according to Eq. (1.1). The lower part of the figure shows the relative impact of the one-loop correction on the relic density compared to micrOMEGAs (second item in the legend). 

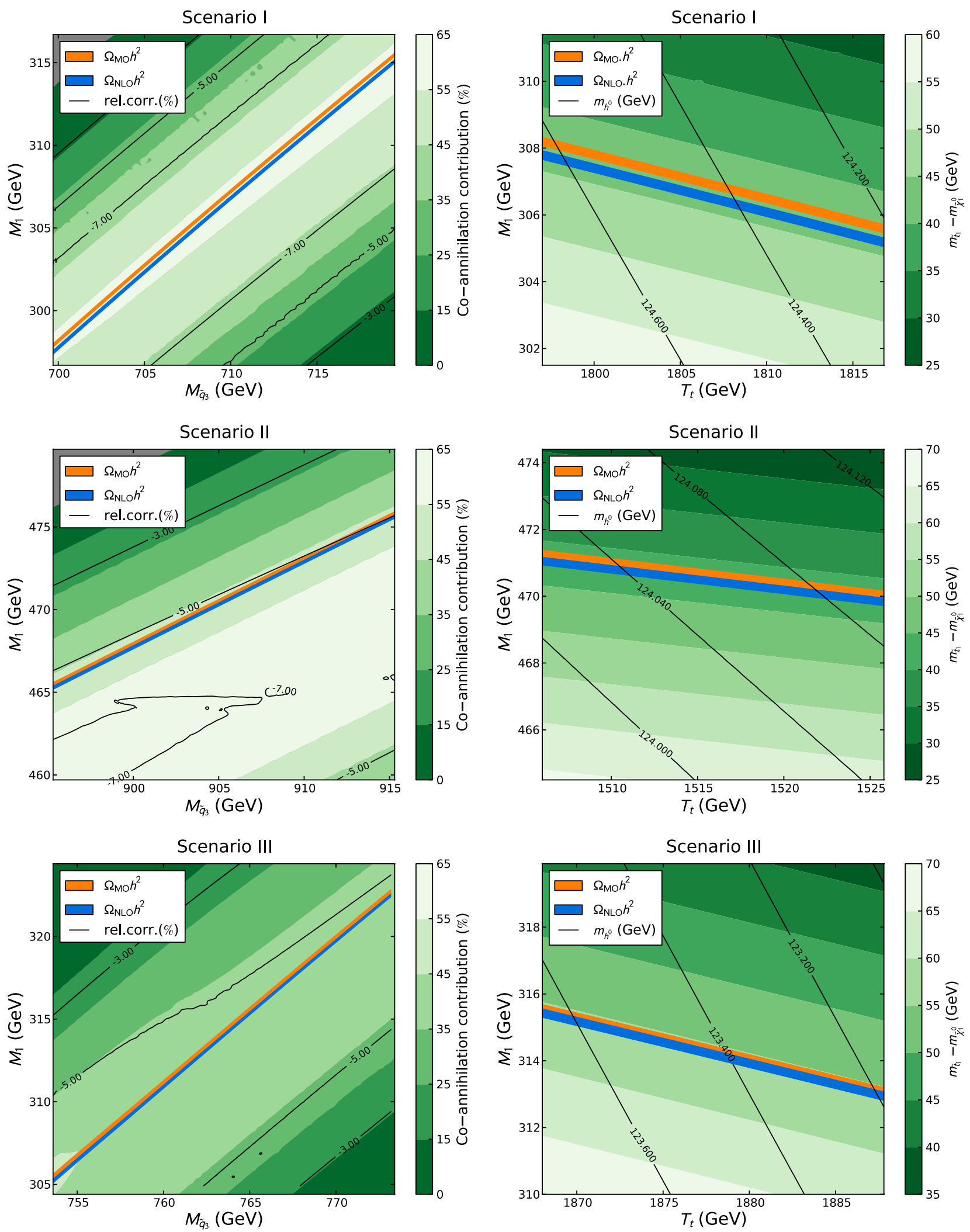

FIG. 13 (color online). WMAP-compatible relic density bands from the default micrOMEGAs calculation (orange) and our one-loop calculation for coannihilation (blue) in the $\left(M_{\tilde{q}_{3}}, M_{1}\right)$ (left) and $\left(T_{t}, M_{1}\right)$ (right) planes. In the plots on the left-hand side the relative contribution of coannihilation processes is shown in green contour, and the relative impact of the one-loop corrections on the relic density in black lines. The plots on the right-hand side show the LSP-NLSP mass difference in green contour, and the lightest neutral Higgs boson mass in black lines. 
micrOMEGAs to the one calculated with the one-loop SUSY-QCD corrections is visible. An interesting feature can be observed by comparing the plots in the second column of Fig. 13 regarding the Higgs mass. Whereas for scenarios I and III the Higgs mass is decreasing with an increasing trilinear coupling parameter, it is the opposite for scenario II. Analyzing Eq. (2.1), where the maximal contribution is obtained from a stop mixing for $\left|X_{t}\right| \sim$ $\sqrt{6} M_{\text {SUSY }}$, this effect becomes clear. In scenario II, we find $\left|X_{t}\right|<\sqrt{6} M_{\text {SUSY }}$ and the Higgs mass grows with increasing $X_{t}$, whereas in the other scenarios $\left|X_{t}\right|>$ $\sqrt{6} M_{\text {SUSY }}$ and the Higgs mass decreases as $X_{t}$ gets larger. In addition, it is interesting that in comparison to the other two scenarios, the preferred region lies in the band where the mass splitting between the neutralino and stop is already around 55-60 GeV.

Studying the three different characteristic scenarios, we saw that the impact of the one-loop corrections on the predicted relic density of dark matter can be more important than the current experimental uncertainty by the WMAP observations. Therefore, it is necessary to take them into account for a theoretical prediction of the neutralino relic density.

\section{CONCLUSIONS}

One of the relevant mechanisms to obtain the observed relic density of dark matter relies on the presence of coannihilation of the dark matter candidate with another particle which is almost degenerate in mass. We have studied this situation within the minimal supersymmetric Standard Model (MSSM), where the dark matter candidate is the lightest of the four neutralinos. More precisely, we have focused on the case of coannihilation with a relatively light stop.

We have demonstrated that the interpretation of a new boson with a mass of about $126 \mathrm{GeV}$ in terms of the lightest Higgs boson within the MSSM favors this situation due to the necessity of an important mass splitting in the stop sector. This results in general in one relatively small mass eigenvalue. If this value is close enough to the neutralino mass, coannihilations are the dominant annihilation channel driving the Boltzmann equation. The important stop mass splitting is mostly realized if the trilinear coupling parameter $T_{t}$ in the stop sector is sizable. This in turn increases the relative importance of the neutralino-stop coannihilation into a top quark and a Higgs boson, which is driven by precisely the same trilinear coupling. Other channels, such as coannihilation into a top (bottom) quark and a $Z(W)$ boson are present, but mostly subdominant.
In order to keep up with the current and future experimental accuracies, a reduction of the theoretical uncertainty is necessary. The main source of uncertainty on the particle physics side comes from the calculation of the (co-)annihilation cross section, which governs the Boltzmann equation and thus the prediction of the dark matter relic density. To this end, we have calculated the coannihilation of a neutralino with a stop into final states containing electroweak gauge or Higgs bosons at one-loop order in SUSY-QCD. In particular, we have defined a renormalization scheme, which can consistently be applied to all neutralino annihilation and coannihilation processes. Infrared singularities are handled using the phase-space slicing method. The present work is complementary to previous publications on radiative corrections to neutralino pair annihilation [11-13] or coannihilation with a stop into a top quark and a gluon or a bottom quark and a $W$ boson [19]. In order to obtain a consistent implementation of all coannihilation processes, including the missing case of a gluon final state will be necessary. This step is, however, postponed to a later publication.

In summary, the impact of the one-loop corrections on the predicted relic density of dark matter can be more important than the current experimental uncertainty by the WMAP observations. The presented corrections are therefore essential in predicting the neutralino relic density for a given parameter point or when extracting SUSY parameters from cosmological measurements. This will become even more important when better limits will be derived from the data of the Planck satellite in a very near future.

\section{ACKNOWLEDGMENTS}

The authors would like to thank A. Freitas, M. Meinecke, and C. Yaguna for helpful discussions, W. Porod for communication regarding the SPheno code, and A. Pukhov for providing us with the necessary functions to implement our results into the micrOMEGAs code. This work is supported by DAAD/EGIDE, Project No. PROCOPE 54366394. The work of J.H. and B.H. (in part) is supported by the Landesexzellenz-Initiative Hamburg "Connecting particles to the cosmos." J.H. acknowledges support from the CMIRA program of the Région Rhône-Alpes and would like to thank the LAPTh for its hospitality during her stays. The work of M. K. is supported by the Helmholtz Alliance for Astroparticle Physics. The work of Q. L. B. is supported by a Ph.D. grant of the French Ministry for Education and Research. Q. L. B. would like to thank the theory group of IPN Lyon for its hospitality. 
[1] E. Komatsu et al. (The WMAP Collaboration), Astrophys. J. Suppl. Ser. 192, 18 (2011).

[2] P. Gondolo and G. Gelmini, Nucl. Phys. B360, 145 (1991).

[3] K. Griest and D. Seckel, Phys. Rev. D 43, 3191 (1991).

[4] J. Edsjo and P. Gondolo, Phys. Rev. D 56, 1879 (1997).

[5] J. Hamann, S. Hannestad, M. S. Sloth, and Y. Y. Y. Wong, Phys. Rev. D 75, 023522 (2007).

[6] A. Arbey and F. Mahmoudi, Phys. Lett. B 669, 46 (2008).

[7] W. Porod, Comput. Phys. Commun. 153, 275 (2003); W. Porod and F. Staub, Comput. Phys. Commun. 183, 2458 (2012).

[8] G. Bélanger, S. Kraml, and A. Pukhov, Phys. Rev. D 72, 015003 (2005).

[9] P. Gondolo, J. Edsjo, P. Ullio, L. Bergstrom, M. Schelke, and E. A. Baltz, J. Cosmol. Astropart. Phys. 07 (2004) 008; P. Gondolo, J. Edsjo, P. Ullio, L. Bergstrom, M. Schelke, E. A. Baltz, T. Bringmann, and G. Duda, http:// www.darksusy.org.

[10] G. Bélanger, F. Boudjema, A. Pukhov, and A. Semenov, Comput. Phys. Commun. 177, 894 (2007); G. Bélanger, F. Boudjema, A. Pukhov, and A. Semenov, Comput. Phys. Commun. 149, 103 (2002).

[11] B. Herrmann and M. Klasen, Phys. Rev. D 76, 117704 (2007).

[12] B. Herrmann, M. Klasen, and K. Kovarik, Phys. Rev. D 79, 061701 (2009).

[13] B. Herrmann, M. Klasen, and K. Kovarik, Phys. Rev. D 80, 085025 (2009).

[14] N. Baro, F. Boudjema, and A. Semenov, Phys. Lett. B 660, 550 (2008).

[15] N. Baro, G. Chalons, and S. Hao, AIP Conf. Proc. 1200, 1067 (2010).

[16] N. Baro, F. Boudjema, G. Chalons, and S. Hao, Phys. Rev. D 81, 015005 (2010).

[17] F. Boudjema, G. Drieu La Rochelle, and S. Kulkarni, Phys. Rev. D 84, 116001 (2011).

[18] A. Chatterjee, M. Drees, and S. Kulkarni, Phys. Rev. D 86, 105025 (2012).

[19] A. Freitas, Phys. Lett. B 652, 280 (2007).

[20] C. Boehm, A. Djouadi, and M. Drees, Phys. Rev. D 62, 035012 (2000).

[21] J. Ellis, K. A. Olive, and Y. Santoso, Astropart. Phys. 18, 395 (2003).

[22] D. Delepine, J.-M. Gérard, R. G. Felipe, and J. Weyers, Phys. Lett. B 386, 183 (1996).

[23] M. Papucci, J. T. Ruderman, and A. Weiler, J. High Energy Phys. 09 (2012) 035.

[24] R. Auzzi, A. Giveon, S. B. Gudnason, and T. Shacham, J. High Energy Phys. 01 (2013) 169.

[25] Z.-H. Yu, X.-J. Bi, Q.-S. Yan, and P.-F. Yin, arXiv:1211.2997.

[26] C. Kilica and B. Tweedie, arXiv:1211.6106.

[27] G. Aad et al. (ATLAS Collaboration), Phys. Lett. B 716, 1 (2012).

[28] S. Chatrchyan et al. (CMS Collaboration), Phys. Lett. B 716, 30 (2012).

[29] G. Aad et al. (ATLAS Collaboration), Report No. ATLASCONF-2012-170.

[30] A. Arbey, M. Battaglia, A. Djouadi, and F. Mahmoudi, arXiv:1211.4004.

[31] H. E. Haber, R. Hempfling, and A. H. Hoang, Z. Phys. C 75, 539 (1997).
[32] M. Badziak, E. Dudas, M. Olechowski, and S. Pokorski, J. High Energy Phys. 07 (2012) 155.

[33] J. A. Aguilar-Saavedra, A. Ali, B. C. Allanach, R. L. Arnowitt, H. A. Baer, J. A. Bagger, C. Balazs, V.D. Barger et al., Eur. Phys. J. C 46, 43 (2006).

[34] J. Beringer et al. (Particle Data Group Collaboration), Phys. Rev. D 86, 010001 (2012).

[35] D. Asner et al. (Heavy Flavor Averaging Group Collaboration), arXiv:1010.1589; online update at http:// www.slac.stanford.edu/xorg/hfag.

[36] https://twiki.cern.ch/twiki/bin/view/AtlasPublic/Combined SummaryPlots.

[37] https://twiki.cern.ch/twiki/bin/view/CMSPublic/Physics ResultsSUS.

[38] G. Passarino and M. J. G. Veltman, Nucl. Phys. B160, 151 (1979).

[39] A. Denner and S. Dittmaier, Nucl. Phys. B844, 199 (2011).

[40] S. Dittmaier, Nucl. Phys. B675, 447 (2003).

[41] T. Hahn, Comput. Phys. Commun. 140, 418 (2001).

[42] R. Mertig, M. Böhm, and A. Denner, Comput. Phys. Commun. 64, 345 (1991).

[43] J. A. M. Vermaseren, arXiv:math-ph/0010025.

[44] http://dmnlo.hepforge.org.

[45] N. Baro and F. Boudjema, Phys. Rev. D 80, 076010 (2009).

[46] S. Heinemeyer, H. Rzehak, and C. Schappacher, Phys. Rev. D 82, 075010 (2010).

[47] K. Kovařík, C. Weber, H. Eberl, and W. Majerotto, Phys. Rev. D 72, 053010 (2005).

[48] K. Melnikov and A. Yelkhovsky, Phys. Rev. D 59, 114009 (1999); A. H. Hoang, Phys. Rev. D 61, 034005 (1999); M. Beneke and A. Signer, Phys. Lett. B 471, 233 (1999); A. A. Penin and A. A. Pivovarov, Nucl. Phys. B549, 217 (1999).

[49] H. Baer, J. Ferrandis, K. Melnikov, and X. Tata, Phys. Rev. D 66, 074007 (2002).

[50] K. G. Chetyrkin, Phys. Lett. B 390, 309 (1997); P. A. Baikov, K. G. Chetyrkin, and J.H. Kuhn, Phys. Rev. Lett. 96, 012003 (2006); K. G. Chetyrkin and A. Kwiatkowski, Nucl. Phys. B461, 3 (1996).

[51] M.S. Carena, D. Garcia, U. Nierste, and C. E. M. Wagner, Nucl. Phys. B577, 88 (2000).

[52] J. Guasch, P. Häfliger, and M. Spira, Phys. Rev. D 68, 115001 (2003).

[53] H. Eberl, K. Hidaka, S. Kraml, W. Majerotto, and Y. Yamada, Phys. Rev. D 62, 055006 (2000).

[54] C. Weber, K. Kovarik, H. Eberl, and W. Majerotto, Nucl. Phys. B776, 138 (2007).

[55] W. T. Giele and E. W. N. Glover, Phys. Rev. D 46, 1980 (1992).

[56] B. W. Harris and J.F. Owens, Phys. Rev. D 65, 094032 (2002).

[57] A. Denner, Fortschr. Phys. 41, 307 (1993).

[58] S. Catani, S. Dittmaier, M. H. Seymour, and Z. Trocsanyi, Nucl. Phys. B627, 189 (2002).

[59] G. 't Hooft and M. J. G. Veltman, Nucl. Phys. B153, 365 (1979).

[60] W. Beenakker, R. Höpker, M. Spira, and P. M. Zerwas, Nucl. Phys. B492, 51 (1997).

[61] T. M. P. Tait, Phys. Rev. D 61, 034001 (1999).

[62] D. Goncalves-Netto, D. Lopez-Val, K. Mawatari, T. Plehn, and I. Wigmore, Phys. Rev. D 87, 014002 (2013).

[63] A. Pukhov, arXiv:hep-ph/0412191. 